\title{
Configuration Structures
}

\author{
R.J. van Glabbeek* \\ Computer Science Department \\ Stanford University \\ Stanford, CA 94305-9045, USA \\ rvg@cs.stanford.edu
}

\author{
G.D. Plotkin* \\ Department of Computer Science \\ University of Edinburgh \\ Edinburgh EH9 3JZ, UK \\ gdp@dcs.ed.ac.uk
}

In this paper the correspondence between safe Petri nets and event structures, due to Nielsen, Plotkin and Winskel, is extended to arbitrary nets without self-loops, under the collective token interpretation. To this end we propose a more general form of event structure, matching the expressive power of such nets. These new event structures and nets are connected by relating both notions with configuration structures, which can be regarded as representations of either event structures or nets that capture their behaviour in terms of action occurrences and the causal relationships between them, but abstract from any auxiliary structure.

A configuration structure can also be considered logically, as a class of propositional models, or-equivalentlyas a propositional theory in disjunctive normal from. Converting this theory to conjunctive normal form is the key idea in the translation of such a structure into a net.

For a variety of classes of event structures we characterise the associated classes of configuration structures in terms of their closure properties, as well as in terms of the axiomatisability of the associated propositional theories by formulae of simple prescribed forms.

\section{Introduction}

The aim of this paper is to connect several models of concurrency, by providing behaviour preserving translations between them.

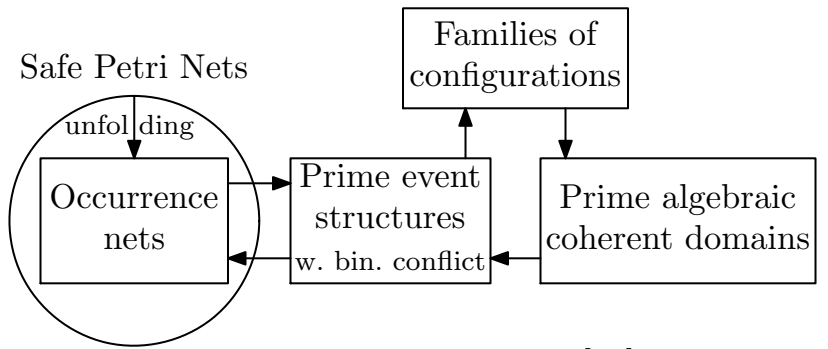

In Nielsen, Plotkin \& Winskel [17] event structures were introduced as a stepping stone between Petri nets and Scott domains. It was established that every safe Petri net can be unfolded into an occurrence net; the occurrence nets are then in correspondence

\footnotetext{
*This work was supported by ONR under grant number N00014-92-J-1974.
}

with event structures; and they in turn correspond bijectively with prime algebraic coherent Scott domains. In WiNSKEL [23] a more general notion of event structure was proposed, corresponding to a more general kind of Scott domain. The event structures from [17] are now called prime event structures with binary conflict.

The translation from event structures to domains passes through a stage of families of configurations of event structures. VAN GLABBEEK \& GolTz [6] found it convenient to use such families as a model of concurrency in their own right. In this context the families were called configuration structures.

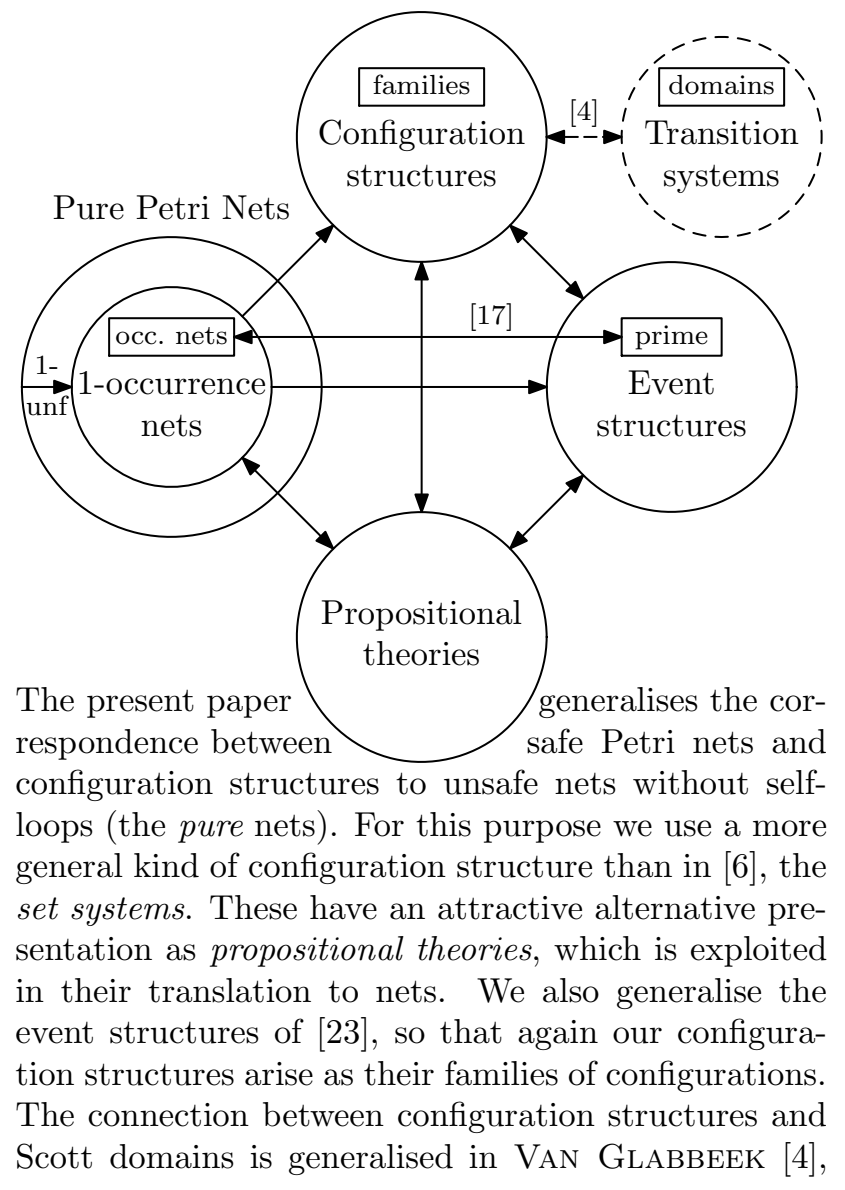


where transition systems are proposed as alternative presentations of domains.

We now consider the models in more detail.

\section{Four models of concurrency}

In this section we present the four models of concurrency mentioned in the introduction, and provide translations between them.

\subsection{Configuration structures}

Definition 1.1 A set system is a pair $\mathrm{C}=\langle E, C\rangle$ with $E$ a set and $C \subseteq \mathcal{P}(E)$ a collection of subsets.

When a set system is used to represent a concurrent system, we call it a (pure) configuration structure. The elements of $E$ are then events and the elements of $C$ configurations. An event represents an occurrence of an action the system may perform; a configuration $x$ represents a state of the system, namely the state in which the events in $x$ have occurred.

Definition 1.2 A configuration $x \in C$ in a configuration structure $\mathrm{C}=\langle E, C\rangle$ is reachable if there is a sequence of events $e_{1}, e_{2}, \ldots, e_{n}$ such that $x=\left\{e_{1}, \ldots, e_{n}\right\}$ and $\forall k \leq n .\left\{e_{1}, \ldots, e_{k}\right\} \in C$. Let $R(\mathrm{C})$ be the set of reachable configurations in $\mathrm{C}$.

A configuration structure is connected if all its configurations are reachable, and if $\mathrm{C}=\langle E, C\rangle$ is any configuration structure, then its reachable part is given by $\mathcal{R}(\mathrm{C})=\langle E, R(\mathrm{C})\rangle$.

For configuration structures representing discrete systems, performing only a finite number of actions in any finite time, one could argue, depending on their precise computational interpretation, that only their reachable parts contain relevant information.

The configuration structures of $[6]$ were required to be nonempty, connected and closed under bounded unions. In the present paper a more expressive kind of configuration structure is considered, not bound by these requirements. Two further generalisations of this model were previously proposed by Pinna \& Poigné [18] and Hoogers, KleiJn \& Thiagarajan [12]. The event automata of [18] and the local event structures of [12] both are configuration structures together with a transition relation between the configurations.

Our configuration structures are, up to isomorphism, the extensional Chu spaces of GUPTA \& PRATT $[11,10,21]$. It was in their work that the idea arose of using the full generality of such structures in modelling concurrency. It should be noted however that the computational interpretation in $[11,10,21]$ differs somewhat from that in $[23,6,18,12,4]$. In particular, in $[11,10,21]$ unreachable configurations may be semantically relevant (cf. the notions of causality and internal choice in $[11,21]$ and the notion of history preserving bisimulation in [10]).

\subsection{Propositional theories}

A set system can also be considered from a logical point of view: $E$ is thought of as a collection of propositions and $C$ as the collection of models. Connecting with the computational point of view, we associate with an event the proposition that it has happened. This point of view is due to Pratt $[11,21]$. We can now represent a set system by the theory $T(C)$ of all valid sentences, those holding in all models; these are the laws of $\mathrm{C}$.

To make this precise, we choose a language: infinitary propositional logic with $E$ as the set of propositional variables, and closed under $\neg$ (negation) and $\bigwedge$ (conjunction of sets of formulae). We make free use of other standard connectives such as $\Rightarrow, \bigvee, \perp, \top$ : they are all definable from $\neg$ and $\bigwedge$.

Definition 1.3 An (infinitary) propositional theory is a pair $\langle E, T\rangle$ with $E$ a set of propositional variables and $T$ a class of infinitary propositional formulae over $E$.

A formula $\phi$ is valid in a set system $\mathrm{C}=\langle E, C\rangle$ iff it is true in all models, that is, elements of $C$; the theory associated to $\mathrm{C}$ is $\mathcal{T}(\mathrm{C})=\langle E, T(C)\rangle$, where $T(\mathrm{C})$ denotes the class of formulae valid in $\mathrm{C}$. Equally, given a propositional theory $\mathrm{T}=\langle E, T\rangle$, its associated set system is $\mathcal{M}(\mathrm{T})=\langle E, M(T)\rangle$, where $M(T)$ is the set of models of $T$, those interpretations making every formula in $T$ true. We say that $\mathrm{T}$ axiomatises $\mathcal{M}(\mathrm{T})$. A formula $\phi$ is a logical consequence of a theory $\mathrm{T}$ if $\phi$ is true in any model of $\mathrm{T}$. Two propositional theories $\mathrm{T}$ and $\mathrm{T}^{\prime}$ are logically equivalent if $\mathcal{M}(\mathrm{T})=\mathcal{M}\left(\mathrm{T}^{\prime}\right)$, i.e. iff they have the same logical consequences.

Proposition 1.1 Let $\mathrm{C}=\langle E, C\rangle$ be a set system. Then $\mathcal{T}(\mathrm{C})$ axiomatises $\mathrm{C}$, i.e. $\mathcal{M}(\mathcal{T}(\mathrm{C}))=\mathrm{C}$.

Proof: The single formula $\bigvee_{X \in C}(\bigwedge X \wedge \neg \bigvee(E-X))$ already constitutes an axiomatisation of $\mathrm{C}$. It is called the disjunctive normal form of $\mathcal{T}(\mathrm{C})$.

Thus $\mathcal{T}$ and $\mathcal{M}$ provide a bijective correspondence between set systems and infinitary propositional theories up to logical equivalence. For any two subsets $X, Y$ of $E$, let the clause $X \Rightarrow Y$ abbreviate the implication $\bigwedge X \Rightarrow \bigvee Y$. Now for any set system $\mathrm{C}=\langle E, C\rangle$, the set of clauses $\{X \Rightarrow(E-X) \mid X \notin C\}$ constitutes another axiomatisation of $\mathrm{C}$. A theory consisting of a set of clauses is said to be in conjunctive normal form. 


\section{$1.3 \quad$ Event structures}

Definition 1.4 An event structure is a pair $\langle E, \vdash\rangle$ with

- $E$ a set of events,

- $\vdash \subseteq \mathcal{P}(E) \times \mathcal{P}(E)$, the enabling relation.

Like a configuration structure, an event structure describes a concurrent system in which the events represent action occurrences. In previous notions of event structure [23, 24], one only had singleton enablings: $\vdash \subseteq \mathcal{P}(E) \times E$. Here we generalise $\vdash$ to a relation between sets of events. As before, the enabling relation places some restrictions on which events can happen when. The idea here is that when $X$ is the set of events that happened so far, an additional set $U$ of events can happen (concurrently) iff every subset of $X \cup U$ is enabled by a set of events that happened before, i.e. a subset of $X$.

In Section 2 we will explain how the event structures above generalise the ones of $[17,23,24]$. In these papers the behaviour of an event structure is formalised by associating to it a family of configurations. However, there are several ways to do so; here we consider the most liberal and the most restrictive variant. A third variant, together with a computational explanation of the notion $S(\mathrm{E})$ below, will appear in Section 2.1 .

Definition 1.5 Let $\mathrm{E}=\langle E, \vdash\rangle$ be an event structure. The set $L(\mathrm{E})$ of left-closed configurations of $\mathrm{E}$ is given by

$$
X \in L(\mathrm{E}) \Leftrightarrow \forall Y \subseteq X . \exists Z \subseteq X . Z \vdash Y
$$

and the set $S(\mathrm{E})$ of secured configurations of E by

$$
X \in S(\mathrm{E}) \Leftrightarrow\left\{\begin{array}{l}
\exists e_{1}, \ldots, e_{n} \in X . X=\left\{e_{1}, \ldots, e_{n}\right\} \\
\wedge \forall k \leq n . \forall Y \subseteq\left\{e_{1}, \ldots, e_{k}\right\} . \\
\exists Z \subseteq\left\{e_{1}, \ldots, e_{k-1}\right\} . Z \vdash Y .
\end{array}\right.
$$

Such a sequence $e_{1}, \ldots, e_{n}$ is called a securing of $X$. The left-closed configuration structure associated to $\mathrm{E}$ is $\mathcal{L}(\mathrm{E})=\langle E, L(\mathrm{E})\rangle$, and likewise $\mathcal{S}(\mathrm{E})=\langle E, S(\mathrm{E})\rangle$ is the secured one.

Two event structures $\mathrm{E}$ and $\mathrm{F}$ are $\mathcal{L}$-equivalent if $\mathcal{L}(\mathrm{E})=\mathcal{L}(\mathrm{F})$ and $\mathcal{S}$-equivalent if $\mathcal{S}(\mathrm{E})=\mathcal{S}(\mathrm{F})$.

For every event structure E we have $S(\mathrm{E}) \subseteq L(\mathrm{E})$. We now introduce a class of event structures $\mathrm{E}$ on which $\mathcal{S}(\mathrm{E})$ can be interpreted as the reachable part of $\mathcal{L}(\mathrm{E})$.

Definition 1.6 An event structure is pure if $X \vdash Y$ only if $X \cap Y=\emptyset$

Proposition 1.2 Let E be a pure event structure. Then $\mathcal{S}(\mathrm{E})=\mathcal{R}(\mathcal{L}(\mathrm{E}))$.
Proof: As $S(\mathrm{E}) \subseteq L(\mathrm{E})$ and $\mathcal{S}(\mathrm{E})$ is always connected we have $S(\mathrm{E})=R(S(\mathrm{E})) \subseteq R(L(\mathrm{E}))$.

Now suppose $x \in R(L(\mathrm{E}))$. Then $x=\left\{e_{1}, \ldots, e_{n}\right\}$ such that $\forall k \leq n$. $\left\{e_{1}, \ldots, e_{k}\right\} \in L(\mathrm{E})$. Take $k \leq n$ and $Y \subseteq\left\{e_{1}, \ldots, e_{k}\right\}$. We must show that there is a $Z \subseteq\left\{e_{1}, \ldots, e_{k-1}\right\}$ with $Z \vdash Y$. Suppose first that $e_{k} \in Y$. Then, since $\left\{e_{1}, \ldots, e_{k}\right\} \in L(\mathrm{E})$, there must be a $Z \subseteq\left\{e_{1}, \ldots, e_{k}\right\}$ with $Z \vdash Y$. As E is pure, $e_{k} \notin Z$, and so $Z \subseteq\left\{e_{1}, \ldots, e_{k-1}\right\}$. Next, suppose instead that $e_{k} \notin Y$. Then, as $\left\{e_{1}, \ldots, e_{k-1}\right\} \in L(\mathrm{E})$, there must be a $Z \subseteq\left\{e_{1}, \ldots, e_{k-1}\right\}$ with $Z \vdash Y$. It follows that $x \in S(\mathrm{E})$.

Hence on pure event structures $\mathcal{L}$-equivalence is finer than $\mathcal{S}$-equivalence. The following example shows that this is strictly so.

Example 1 Take as events the set $\mathbb{Q}$ of rational numbers and define $\vdash$ by $\emptyset \vdash X$ for any $X$ with $|X| \neq 1$, and $X \vdash\{e\}$ iff $X=\{d \in \mathbb{Q} \mid d<e\}$. We have $S(\mathrm{E})=\{\emptyset\}$, whereas $L(\mathrm{E})$ additionally contains representatives of all reals as well as extra copies of the rationals and $\mathbb{Q}$ itself (infinity). If $\mathrm{E}^{\prime}$ is $\langle\mathbb{Q},\{(\emptyset, \emptyset)\}\rangle$ we have $\mathcal{S}(\mathrm{E})=\mathcal{S}\left(\mathrm{E}^{\prime}\right)$, yet $\mathcal{L}(\mathrm{E}) \neq \mathcal{L}\left(\mathrm{E}^{\prime}\right)$.

We now show that any configuration structure can be obtained as the left-closed configuration structure associated to a pure event structure, and hence any connected configuration structure can be obtained as the secured configuration structure associated to a pure event structure.

Definition 1.7 Let $\mathrm{C}=\langle E, C\rangle$ be a configuration structure. The event structure associated to $\mathrm{C}$ is $\mathcal{E}(\mathrm{C})=\langle E, \vdash\rangle$, with $X \vdash Y$ iff $X \cap Y=\emptyset \wedge X \cup Y \in C$.

Theorem 1 Let $\mathrm{C}$ be a configuration structure. Then $\mathcal{L}(\mathcal{E}(\mathrm{C}))=\mathrm{C}$.

Proof: Let $\mathrm{C}=\langle E, C\rangle$ and $\mathcal{E}(\mathrm{C})=\langle E, \vdash\rangle$. Suppose $x \in C$. For any $Y \subseteq x$ take $Z=x-Y$. Then $Z \subseteq x$ and $Z \vdash Y$. So $x \in \mathcal{L}(\mathcal{E}(\mathrm{C}))$. Conversely, suppose $X \in \mathcal{L}(\mathcal{E}(\mathrm{C}))$. Then there is a $Z \subseteq X$ such that $Z \vdash X$. By construction, $X=Z \cup X \in C$.

Corollary 1 Let $\mathrm{C}$ be a connected configuration structure. Then $\mathcal{S}(\mathcal{E}(\mathrm{C}))=\mathrm{C} \quad(=\mathcal{L}(\mathcal{E}(\mathrm{C})))$.

Proof: $\mathcal{S}(\mathcal{E}(\mathrm{C}))=\mathcal{R}(\mathcal{L}(\mathcal{E}(\mathrm{C})))=\mathcal{R}(\mathrm{C})=\mathrm{C}$.

Hence, $\mathcal{E}$ and $\mathcal{L}$ provide a bijective correspondence between configuration structures and (pure) event structures up to $\mathcal{L}$-equivalence, and $\mathcal{E}$ and $\mathcal{S}$ between connected configuration structures and (pure) event structures up to $\mathcal{S}$-equivalence.

The following shows that Proposition 1.2 does not extend to impure event structures. For those, $\mathcal{L}$ and $\mathcal{S}$ represent mutually inconsistent interpretations. 
Example 2 Let $\mathrm{E}=\langle\{e\},\{(\emptyset, \emptyset),(\{e\},\{e\})\}\rangle$. Then $\mathcal{L}(\mathrm{E})=\langle\{e\},\{\emptyset,\{e\}\}\rangle$, whereas $\mathcal{S}(\mathrm{E})=\langle\{e\},\{\emptyset\}\rangle$. Both configuration structures are connected.

Let $\mathrm{E}^{\prime}=\langle\{e\},\{(\emptyset, \emptyset),(\emptyset,\{e\})\}\rangle$. Then we have $\mathcal{L}(\mathrm{E})=\mathcal{L}\left(\mathrm{E}^{\prime}\right)$ but $\mathcal{S}(\mathrm{E}) \neq \mathcal{S}\left(\mathrm{E}^{\prime}\right)$.

However, under either interpretation the impure event structures are redundant: for every event structure there exists a pure one with the same configurations. Obviously, which one depends on whether the leftclosed or the secured configurations are to be preserved.

Corollary 2 For any event structure $\mathrm{E}$ there is a pure event structure $\mathrm{E}_{\mathcal{L}}$ with $\mathcal{L}\left(\mathrm{E}_{L}\right)=\mathcal{L}(\mathrm{E})$, and a pure event structure $\mathrm{E}_{\mathcal{S}}$ with $\mathcal{S}\left(\mathrm{E}_{S}\right)=\mathcal{S}(\mathrm{E})$.

Proof: Take $\mathrm{E}_{\mathcal{L}}=\mathcal{E}(\mathcal{L}(\mathrm{E}))$ and $\mathrm{E}_{\mathcal{S}}=\mathcal{E}(\mathcal{S}(\mathrm{E}))$.

A structure $\mathrm{E}_{\mathcal{L}}=\left\langle E, \vdash_{\mathcal{L}}\right\rangle$ can also be directly obtained by putting $\vdash_{\mathcal{L}}=\{(X-Y, Y) \mid X \vdash Y\}$.

\section{Event structures vs. propositional theories}

With any event structure $\mathrm{E}=\langle E, \vdash\rangle$ we associate the propositional theory $\mathcal{T}(\mathrm{E})=\langle E, T(\mathrm{E})\rangle$, where

$$
T(\mathrm{E})=\{X \Rightarrow \bigvee\{\bigwedge Y \mid Y \vdash X\} \mid X \subseteq E\} .
$$

This logical view of event structures corresponds exactly with their left-closed interpretation:

Proposition 1.3 $\mathcal{M}(\mathcal{T}(\mathrm{E}))=\mathcal{L}(\mathrm{E})$ for any event structure E.

Proof: Immediate from the definitions.

Likewise, with any propositional theory $\mathrm{T}=\langle E, T\rangle$ in conjunctive normal form we associate the event structure $\mathcal{E}(\mathrm{T})=\left\langle E, \vdash_{\mathrm{T}}\right\rangle$, where

$$
X \vdash_{\mathrm{T}} Y \Leftrightarrow \forall Z .((Y \Rightarrow Z) \in T \Rightarrow X \cap Z \neq \emptyset) .
$$

Proposition $1.4 \mathcal{L}(\mathcal{E}(\mathrm{T}))=\mathcal{M}(\mathrm{T})$ for any theory $\mathrm{T}$ in conjunctive normal form.

Proof: Let $x \in \mathcal{M}(\mathrm{T})$ and $Y \subseteq x$ It suffices to show that $x \vdash_{\mathrm{T}} Y$. Let $Z \subseteq E$ be such that $(Y \Rightarrow Z) \in T$. As $Y \Rightarrow Z$ is true in $x$ we have $Z \cap x \neq \emptyset$. It follows that $x \in \mathcal{L}(\mathcal{E}(\mathrm{T}))$.

Now let $x \in \mathcal{L}(\mathcal{E}(\mathrm{T}))$ and let $(Y \Rightarrow Z) \in T$. We have to show that $Y \Rightarrow Z$ is true in $x$. In case $Y \nsubseteq x$ this is trivially the case, so suppose $Y \subseteq x$. Then there must be a $W \subseteq x$ with $W \vdash_{\mathrm{T}} Y$, hence $W \cap Z \neq \emptyset$. It follows that $x \cap Z \neq \emptyset$, which had to be shown.

Thus $\mathcal{T}$ and $\mathcal{E}$ provide a bijective correspondence between event structures up to $\mathcal{L}$-equivalence and propositional theories up to logical equivalence.

\subsection{Rooted and finitary structures}

In order to translate between the models of concurrency seen so far and Petri nets, we have to restrict to structures that are rooted and finitary.

Definition 1.8 A configuration structure $\mathrm{C}=\langle E, C\rangle$ is rooted if $\emptyset \in C$. A propositional theory is rooted if it has no clause of the form $\emptyset \Rightarrow X$ as a logical consequence. An event structure $\mathrm{E}=\langle E, \vdash\rangle$ is rooted if $\emptyset \vdash \emptyset$.

Proposition 1.5 If $\mathrm{C}$ is rooted, then so are $\mathcal{T}(\mathrm{C})$ and $\mathcal{E}(\mathrm{C})$. If $\mathrm{T}$ is rooted, then so are $\mathcal{M}(\mathrm{T})$ and $\mathcal{E}(\mathrm{T})$. If $\mathrm{E}$ is rooted, then so are $\mathcal{L}(\mathrm{E}), \mathcal{S}(\mathrm{E})$ and $\mathcal{T}(\mathrm{E})$.

Proof: Straightforward.

Definition 1.9 A configuration structure is finitary if all its configurations are finite. A propositional theory is finitary if, for every infinite $X \subseteq E$, it has the clause $X \Rightarrow \emptyset$ as a logical consequence. An event structure $\langle E, \vdash\rangle$ is finitary if $X \vdash Y$ only holds for finite sets $Y$.

Proposition 1.6 If $\mathrm{C}$ is finitary, then so are $\mathcal{T}(\mathrm{C})$ and $\mathcal{E}(\mathrm{C})$. If $\mathrm{T}$ is finitary, then so are $\mathcal{M}(\mathrm{T})$ and $\mathcal{E}(\mathrm{T})$. If $\mathrm{E}$ is finitary, then so are $\mathcal{L}(\mathrm{E}), \mathcal{S}(\mathrm{E})$ and $\mathcal{T}(\mathrm{E})$.

Proof: Straightforward.

Note that connected configuration structures are always finitary, and are either rooted or have an empty set of configurations.

\subsection{Petri nets}

\section{Definition 1.10}

A Petri net is a tuple $\mathrm{N}=\langle S, T, F, I\rangle$ with

- $S$ and $T$ two disjoint sets of places and transitions,

- $F:(S \times T \cup T \times S) \rightarrow \mathbb{N}$, the flow relation,

- and $I: S \rightarrow \mathbb{N}$, the initial marking.

Petri nets are pictured by drawing the places as circles and the transitions as boxes. For $x, y \in S \cup T$ there are $F(x, y)$ arcs from $x$ to $y$. A net is said to be without arcweights if the range of $F$ is $\{0,1\}$.

When a Petri net represents a concurrent system, a global state of such a system is given as a marking, which is a multiset over $S$, i.e. a function $M \in \mathbb{N}^{S}$. Such a state is depicted by placing $M(s)$ dots (tokens) in each place $s$. The initial state is given by the marking $I$. In order to describe the behaviour of a net, we describe the step transition relation between markings.

Definition 1.11 For two multisets $M$ and $N$ over $S$, or more general for functions $M, N \in \mathbb{Z}^{S}$, write $M \leq$ $N$ if $M(s) \leq N(s)$ for all $s \in S ; M+N \in \mathbb{Z}^{S}$ is 
the function given by $(M+N)(s)=M(s)+N(s)$, and $0 \in \mathbb{N}^{S}$ the one with $0(s)=0$ for all $s \in S$; $M-N \in \mathbb{Z}^{S}$ is given by $(M-N)(s)=M(s)-N(s)$.

A multiset $M$ over $S$ is finite if $\{s \in S \mid M(s)>0\}$ is finite. A multiset $M \in \mathbb{N}^{S}$ with $M(s) \leq 1$ for all $s \in S$ is identified with the set $\{s \in S \mid M(s)=1\}$.

Note that for multisets $M$ and $N$, the function $M-N$ need not be a multiset.

Definition 1.12 For a finite multiset $U: T \rightarrow \mathbb{N}$ of transitions in a Petri net, let ${ }^{\bullet} U, U^{\bullet}: S \rightarrow \mathbb{N}$ be the multisets of pre- and postplaces of $U$, given by

- $U(s)=\sum_{t \in T} F(s, t) \cdot U(t)$ and $U^{\bullet}(s)=\sum_{t \in T} U(t) \cdot F(t, s)$

for $s \in S$. $U$ is enabled under a marking $M$ if $\bullet U \leq M$. In that case $U$ can fire under $M$, yielding the marking $M^{\prime}=M-\bullet U+U^{\bullet}$, written $M \stackrel{U}{\longrightarrow} M^{\prime}$.

A chain $I \stackrel{U_{1}}{\longrightarrow} M_{1} \stackrel{U_{2}}{\longrightarrow} \cdots \stackrel{U_{n}}{\longrightarrow} M_{n}$ is called a firing sequence. A marking $M$ is reachable if there is such a sequence ending in $M=M_{n}$.

If a multiset $U$ of transitions fires, for every transition $t$ in $U$ and every arc from a place $s$ to $t$, a token moves along that arc from $s$ to $t$. These tokens are consumed by the firing, but also new tokens are created, namely one for every outgoing arc of $t$. These end up in the places at the end of those arcs. If $t$ occurs several times in $U$, all this happens several times (in parallel) as well. The firing of $U$ is only possible if there are sufficiently many tokens in the preplaces of $U$ (the places where the incoming arcs come from).

As for event structures, the behaviour of a net can be explained by associating to it a family of configurations. Again we consider two ways of doing so, analogously to the left-closed and secured configurations of event structures, respectively.

Definition 1.13 A configuration of a net is any finite multiset $X$ of transitions with the property that the function $M_{X}: S \rightarrow \mathbb{Z}$ given by $M_{X}=I-\bullet X+X \bullet$ is a marking (i.e. $M_{X} \geq 0$ ). The firing relation between markings induces one between the configurations of a net:

$$
X \longrightarrow Y \Leftrightarrow\left(X \leq Y \wedge M_{X} \stackrel{Y-X}{\longrightarrow} M_{Y}\right) .
$$

Let $L(\mathrm{~N})$ denote the set of configurations of the net $\mathrm{N}$.

Note that if $X$ is a configuration and $M_{X} \stackrel{U}{\longrightarrow} M^{\prime}$ then $X+U$ is a configuration and $M^{\prime}=M_{X+U}$. So if $I \stackrel{U_{1}}{\longrightarrow} M_{1} \stackrel{U_{2}}{\longrightarrow} \cdots \stackrel{U_{n}}{\longrightarrow} M_{n}$ is a firing sequence, then $X=U_{1}+\cdots+U_{n}$ is a configuration and $M_{n}=M_{X}$.
Definition 1.14 The set $S(\mathrm{~N})$ of secured configurations of a net $\mathrm{N}$ consists of the multisets $\sum_{i=1}^{n} U_{n}$ such that there is a firing sequence

$$
I \stackrel{U_{1}}{\longrightarrow} M_{1} \stackrel{U_{2}}{\longrightarrow} \cdots \stackrel{U_{n}}{\longrightarrow} M_{n} .
$$

By the above, we have $S(\mathrm{~N}) \subseteq L(\mathrm{~N})$.

Next we will determine which nets can be described by means of set systems.

Definition 1.15 A 1-occurrence net is a net in which every configuration is a set.

This implies that any transition can fire at most once, i.e. in every firing sequence $M_{0} \stackrel{U_{1}}{\longrightarrow} \cdots \stackrel{U_{n}}{\longrightarrow} M_{n}$ the multisets $U_{1}, \ldots, U_{n}$ are sets and disjoint. If $\mathrm{N}=$ $\langle S, T, F, I\rangle$ is a 1 -occurrence net, then $\langle T, L(\mathrm{~N})\rangle$ is a set system.

In general the induced firing relation of a net is not determined by its set of configurations. The 1occurrence nets $\mathrm{P}$ and $\mathrm{Q}$ below have very different behaviour: in $\mathrm{P}$ the transitions $d$ and $e$ can be done in parallel (there is a transition $\emptyset \longrightarrow\{d, e\}$ ), whereas in $\mathrm{Q}$ there is mutual exclusion. Yet their configura-
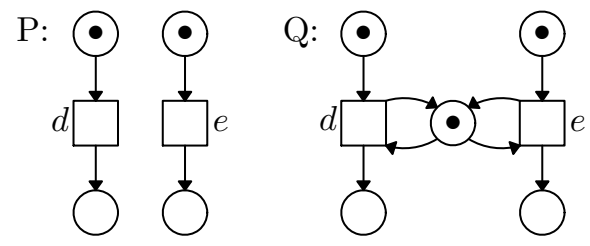

tions are the same: $L(\mathrm{P})=L(\mathrm{Q})=S(\mathrm{P})=S(\mathrm{Q})=$ $\{\emptyset,\{d\},\{e\},\{d, e\}\}$. Therefore it is not a good idea to equate every 1-occurrence net $\mathrm{N}=\langle S, T, F, I\rangle$ with its configuration structure $\langle T, L(\mathrm{~N})\rangle$. However, for the subclass of pure Petri nets this can be justified:

Definition 1.16 A net $\mathrm{N}=\langle S, T, F, I\rangle$ is pure if there is no $s$ in $S$ and $t$ in $T$ with $F(s, t)>0$ and $F(t, s)>0$, i.e. if it is without self-loops.

Proposition 1.7 In a pure net N we have $X \longrightarrow Y$ iff $X \leq Y \wedge \forall Z(X \subset Z \subset Y \Rightarrow Z \in L(\mathrm{~N}))$ for all $X, Y$ in $L(\mathrm{~N})$.

Proof: "only if": Let $X \longrightarrow Y$ for $X, Y \in L(\mathrm{~N})$. Then $M_{X}=I-\bullet X+X \bullet \geq 0$ and $Y-X$ is enabled under $M_{X}$, i.e. $\bullet(Y-X) \leq M_{X}$. Now let $X \subset Z \subset Y$. Then $\cdot(Z-X) \leq \cdot(Y-X) \leq M_{X}$, so $M_{Z}=$ $I-\bullet Z+Z \bullet=I-\bullet X+X \bullet-\bullet(Z-X)+(Z-X) \bullet=$ $M_{X}-\bullet(Z-X)+(Z-X)^{\bullet} \geq 0+(Z-X)^{\bullet} \geq 0$, i.e. $Z$ is a configuration of N. Note that for this direction pureness is not needed.

"if": Suppose $X \in L(\mathrm{~N})$ and $X \leq Y$, but $X \nrightarrow Y$. Then $Y-X$ is not enabled under $M_{X}$, i.e. there is a 
place $s \in S$, such that $\bullet(Y-X)(s)>M_{X}(s)$. Let $U$ be the multiset of those transitions $t$ in $Y-X$ for which $F(s, t)>0$. Then ${ }^{\bullet} U(s)=\bullet(Y-X)(s)>M_{X}(s)$. As $\mathrm{N}$ is pure, for all those transitions $t$ we have $F(t, s)=0$, and therefore $U^{\bullet}(s)=0$. Hence $M_{(X+U)}(s)=M_{X}(s)-\bullet U(s)+U^{\bullet}(s)<0$, i.e. $X+U \notin L(\mathrm{~N})$. Yet $X \subseteq(X+U) \subseteq Y$.

It follows that for a pure net the induced firing relation is completely determined by its set of configurations.

Definition 1.17 Let $\mathrm{N}=\langle S, T, F, I\rangle$ be a pure 1occurrence net. Its associated configuration structure $\mathcal{L}(\mathrm{N})$ is $\langle T, L(N)\rangle$. Two such nets $\mathrm{N}$ and $\mathrm{N}^{\prime}$ are configuration equivalent - written $\mathrm{N}=\mathcal{L} \mathrm{N}^{\prime}$-if $\mathcal{L}(\mathrm{N})=\mathcal{L}\left(\mathrm{N}^{\prime}\right)$.

Similarly, one could define the associated secured configuration structure $\mathcal{S}(\mathrm{N})$ of $\mathrm{N}$. Using Proposition 1.7 it follows that again $\mathcal{S}(\mathrm{N})$ is the reachable part of $\mathcal{L}(\mathrm{N})$.

Corollary 3 Let $\mathrm{N}=\langle S, T, F, I\rangle$ be a pure 1-occurrence net. Then $\mathcal{S}(\mathrm{N})=\mathcal{R}(\mathcal{L}(\mathrm{N}))$.

The configuration structure associated to a Petri net is always finitary and rooted. We now proceed to show that every such configuration structure can be obtained as the image of a pure 1-occurrence net.

Definition 1.18 Let $\mathrm{T}=\langle E, T\rangle$ be a finitary rooted propositional theory in conjunctive normal form. We define the associated Petri net $\mathcal{N}(\mathrm{T})$ as follows. As transitions of the net we take the events of the configuration structure. For every transition we add one place, containing one initial token, that has no incoming arcs, and with its only outgoing arc going to that transition. These 1-occurrence places make sure that every transition fires at most once. For every clause $X \Rightarrow Y$ in $T$ with $X$ finite, we introduce a place in the net. This place has outgoing arcs to each of the transitions in $X$, and incoming arcs from each of the places in $Y$. Let $n$ be the cardinality of $X$. As $\mathrm{T}$ is rooted, $n \neq 0$. We finish the construction by putting $n-1$ initial tokens in the created place:

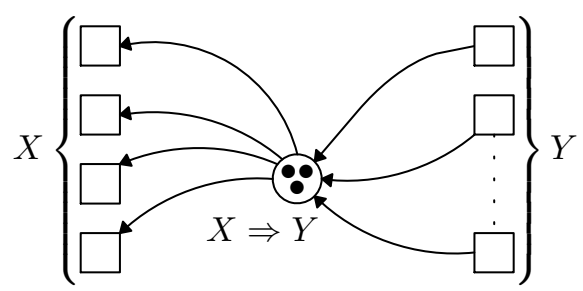

The place belonging to the clause $X \Rightarrow Y$ does not place any restrictions on the firing of the first $n-1$ transitions in $X$. However, the last one can only fire after an extra token arrives in the place. This can happen only if one of the transitions in $Y$ fires first. The firing of more transitions in $Y$ has no adverse effects, as each of the transitions in $X$ can fire only once. Thus this place imposes the same restriction on the occurrence of events as the corresponding clause.

Theorem 2 Let $\mathrm{T}$ be a finitary rooted propositional theory in conjunctive normal form. Then

$$
\mathcal{L}(\mathcal{N}(\mathrm{T}))=\mathcal{M}(\mathrm{T})
$$

Proof: $Z \in L(\mathcal{N}(\mathrm{T}))$ iff $Z$ is finite and $M_{Z}(s) \geq 0$ for any place $s$. We have $M_{Z}(s) \geq 0$ for all 1-occurrence places $s$ exactly when no transition fires twice in $Z$, i.e. when $Z$ is a set. For a place $s$ belonging to the clause $X \Rightarrow Y$ we have $M_{Z}(s) \geq 0$ iff either one of the transitions in $Y$ has fired, or not all of the transitions in $X$ have fired, i.e. when $X \Rightarrow Y$ holds in $Z$, seen as a model of propositional logic. As $\mathrm{T}$ is finitary, all its models will be finite; furthermore, the clauses $X \Rightarrow Y$ of $\mathrm{T}$ with $X$ infinite surely hold in any finite configuration $Z$. Thus, $Z \in L(\mathcal{N}(\mathrm{T}))$ iff $Z$ is a model of $\mathrm{T}$.

The net $\mathcal{N}(\mathrm{T})$ is always without arcweights. Now we examine under which circumstances it is pure.

Definition 1.19 A propositional theory in conjunctive normal form is pure if it only contains clauses $X \Rightarrow Y$ with $X \cap Y=\emptyset$.

Clearly every propositional theory is logically equivalent to a pure one; thus a finitary rooted configuration structure can be axiomatised by a finitary rooted pure propositional theory. Now note that for such a theory $\mathrm{T}$ the net $\mathcal{N}(\mathrm{T})$ is pure as well. It follows that

Corollary 4 For every finitary rooted configuration structure there exists a pure 1-occurrence net without arcweights with the same configurations.

Thus there exists a bijective correspondence between finitary rooted configuration structures and pure 1occurrence nets up to configuration equivalence. Moreover, every pure 1-occurrence net is configuration equivalent to a pure 1-occurrence net without arcweights.

\section{From nets to theories and event structures}

Let $\mathrm{N}=\langle S, E, F, I\rangle$ be a 1-occurrence net. For any place $s \in S$ let $s^{\bullet}=\{t \in E \mid F(s, t)>0\}$ be its set of posttransitions and $\bullet=\{t \in E \mid F(t, e)>0\}$ its set of pretransitions. For any set $Y \subseteq s^{\bullet}$ of posttransitions of $s,{ }^{\bullet} Y(s)$ is the number of tokens needed in place $s$ 
for all transitions in $Y$ to fire, so ${ }^{\bullet} Y(s)-I(s)$ is the number of tokens that have to arrive in place $s$ before all transitions in $Y$ can fire. Furthermore, for any $n \in$ $\mathbb{N}$, let ${ }^{n} s=\left\{X \subseteq{ }^{\bullet} s \mid X^{\bullet}(s) \geq n\right\}$ be the collection of sets $X$ of pretransitions of $s$, such that if all transitions in $X$ fire, at least $n$ tokens will arrive in $s$. Write ${ }^{Y}$ for ${ }^{\bullet} Y(s)-I(s) s$. One of the sets of transitions in ${ }^{S}$ has to fire entirely before all transitions in $Y$ can fire.

The formula $\varphi_{s}^{n}=\bigvee_{X \in n_{s}} \bigwedge X$ expresses which transitions need to fire for $n$ tokens to arrive in place $s$. The formula $\bigwedge Y \Rightarrow \dot{\varphi}_{s} Y(s)-I(s)$ expresses that one of the sets of transitions in ${ }^{Y} S$ has to fire entirely before all transitions in $Y$ can fire. The propositional theory associated to $\mathrm{N}$ is defined as $\mathcal{T}(\mathrm{N})=\langle E, T(\mathrm{~N})\rangle$, where $T(\mathrm{~N})$ consists of all formulas $\bigwedge Y \Rightarrow \dot{\varphi}_{s} Y(s)-I(s)$ with $s \in S$ and $Y \subseteq s^{\bullet}$. It follows that

Proposition 1.8 $\mathcal{M}(\mathcal{T}(\mathrm{N}))=\mathcal{L}(\mathrm{N})$ for any pure 1occurrence net $\mathrm{N}$.

For any set of transitions $Y \subseteq E$, let $S_{Y}$ be the set of places $s$ with $Y \subseteq s^{\bullet}$ and ${ }^{\bullet} Y(s)-I(s)>0$. Now write $X \vdash_{\mathrm{N}} Y$ whenever $X=\bigcup_{s \in S_{Y}} X_{s}$ with $X_{s} \in Y_{s}$. The event structure associated to $\mathrm{N}$ is defined as $\mathcal{E}(\mathrm{N})=$ $\left\langle E, \vdash_{\mathrm{N}}\right\rangle$.

Proposition 1.9 Let $\mathrm{N}$ be a pure 1-occurrence net. Then $\mathcal{L}(\mathcal{E}(\mathrm{N}))=\mathcal{L}(\mathrm{N})$ and $\mathcal{S}(\mathcal{E}(\mathrm{N}))=\mathcal{S}(\mathrm{N})$.

Proof: Straightforward.

\section{Other brands of event structures}

Event structures have been introduced in NIELSEN, Plotkin \& Winskel [17] as triples $\langle E, \leq, \#\rangle$, in Winskel [23] as triples $\langle E, C o n, \vdash\rangle$ and $\langle E$, Con,$\leq\rangle$, and in WINSKEL [24] as triples $\langle E, \#, \vdash\rangle$ and $\langle E, \#, \leq\rangle$. Here we will explain how our event structures generalise these previous proposals. The components \#, Con, $\vdash$ and $\leq$ that occur in the triples mentioned above can be defined in terms of our event structures as follows.

Definition 2.1 Let $\mathrm{E}=\langle E, \vdash\rangle$ be an event structure. Two events $d, e \in X$ are in conflict, written $d \# e$, if

$$
\neg \exists Z . Z \vdash\{d, e\} .
$$

A finite set of events $X \subseteq_{f i n} E$ is consistent or conflictfree, written $\operatorname{Con}(X)$, if

$$
\forall Y \subseteq X . \exists Z \subseteq E . Z \vdash X .
$$

For $X \subseteq_{\text {fin }} E$ and $e \in E$, write $X \vdash^{\prime} e$ for

$$
\operatorname{Con}(X) \wedge \exists Y \subseteq X . Y \vdash\{e\} .
$$

The causality relation $\prec \subseteq E \times E$ is given by

$$
d \prec e \Leftrightarrow \forall X .(X \vdash\{e\} \Rightarrow d \in X) .
$$

Let $\leq$ be the reflexive and transitive closure of $\prec$.

It would be possible to extend the definition of Con to infinite sets of events, and likewise for $\vdash$. This would allow a better formulation of the forthcoming Propositions 2.1-2.3. The reason to stick to the definitions above is to stay as close as possible to [23, 24].

The next definition gives various properties of our event structures which, in suitable combinations, determine subclasses corresponding to the various event structures in $[17,23,24]$.

Definition 2.2 An event structure $\mathrm{E}=\langle E, \vdash\rangle$ is

- singular if $X \vdash Y \Rightarrow X=\emptyset \vee|Y|=1$,

- conjunctive if $X_{i} \vdash Y(i \in I \neq \emptyset) \Rightarrow \bigcap_{i \in I} X_{i} \vdash Y$,

- locally conjunctive if $X_{i} \vdash Y$ (for $\left.i \in I \neq \emptyset\right) \wedge$ $\exists Z . Z \vdash \bigcup_{i \in I} X_{i} \cup Y \Rightarrow \bigcap_{i \in I} X_{i} \vdash Y$,

- $\mathcal{S}$-irredundant if every event occurs in a secured configuration, i.e. $E=\bigcup_{x \in S(\mathrm{E})} x$,

- $\mathcal{L}$-irredundant if every event occurs in a left-closed configuration, i.e. $E=\bigcup_{x \in L(\mathrm{E})} x$,

- and cycle-free if there is no chain

$$
e_{0} \prec e_{1} \prec \cdots \prec e_{n} \prec e_{0}
$$

and has

- finite causes if $X \vdash Y \Rightarrow X$ finite,

- finite conflict if $X$ infinite $\Rightarrow \emptyset \vdash X$

- and binary conflict if $|X|>2 \Rightarrow \emptyset \vdash X$.

The event structures of $[17,23,24]$ all correspond to event structures in our sense that are rooted, singular and with finite conflict. The event structures given as triples involving \# even have binary conflict, the ones involving $\vdash$ have finite causes, and the ones involving $\leq$ are conjunctive, $\mathcal{L}$-irredundant and cycle-free. The event structures of $[23,24]$ that involve $\leq$ are moreover $\mathcal{S}$-irredundant, a property that implies $\mathcal{L}$ irredundancy and cycle-freeness. The requirement of stability in $[23,24]$ corresponds to our notion of local conjunctivity.

For the singular event structures E, the enabling relation consists of two parts: enablings of the form $\emptyset \vdash Y$, and enablings of the form $X \vdash\{e\}$. When $\mathrm{E}$ has finite conflict, the first part can be fully expressed in terms of Con, at least to the extend in which it determines which sets of events are configurations. When $\mathrm{E}$ has finite causes, the second part can similarly be expressed in terms of $\vdash^{\prime}$. One obtains the following. 
Proposition 2.1 Let E be a singular event structure with finite causes and finite conflict. Then

$$
X \in L(\mathrm{E}) \Leftrightarrow\left\{\begin{array}{l}
\forall Y \subseteq \text { fin } X . C o n(Y) \wedge \\
\forall e \in X . \exists Z \subseteq X . Z \vdash^{\prime} e
\end{array}\right.
$$

and

$$
X \in S(\mathrm{E}) \Leftrightarrow\left\{\begin{array}{l}
\operatorname{Con}(X) \wedge \\
\exists e_{1}, \ldots, e_{n} \in X . X=\left\{e_{1}, \ldots, e_{n}\right\} \wedge \\
\forall k \leq n .\left\{e_{1}, \ldots, e_{k-1}\right\} \vdash^{\prime} e_{k} .
\end{array}\right.
$$

It follows that such structures can alternatively be represented as triples $\langle E$, Con,$\vdash\rangle$ with $\operatorname{Con} \subseteq \mathcal{P}_{\text {fin }}(E)$ and $\vdash \subseteq C$ on $\times E$, as are the structures of [23].

When E moreover is rooted and with binary conflict, Con can be fully expressed in terms of \#.

Proposition 2.2 Let E be a rooted, singular event structure with finite causes and binary conflict. Then

and

$$
X \in L(\mathrm{E}) \Leftrightarrow\left\{\begin{array}{l}
\forall d, e \in X . \neg(d \# e) \wedge \\
\forall e \in X . \exists Z \subseteq X . Z \vdash^{\prime} e
\end{array}\right.
$$

$$
X \in S(\mathrm{E}) \Leftrightarrow\left\{\begin{array}{l}
\forall d, e \in X . \neg(d \# e) \wedge \\
\exists e_{1}, \ldots, e_{n} \in X . X=\left\{e_{1}, \ldots, e_{n}\right\} \wedge \\
\forall k \leq n .\left\{e_{1}, \ldots, e_{k-1}\right\} \vdash^{\prime} e_{k} .
\end{array}\right.
$$

It follows that such event structures can alternatively be represented as triples $\langle E, \#, \vdash\rangle$ with $\# \subseteq E \times E$ and $\vdash \subseteq$ Con $\times E$, as are the structures of [24].

When $d \leq e$, any configuration containing $e$ also contains $d$. When $\mathrm{E}=\langle E, \vdash\rangle$ is conjunctive, for any consistent event $e \in E$ there is a smallest set $X \subseteq E$ with $X \vdash e$. Therefore the part of the enabling relation consisting of enablings $X \vdash e$ is in essence completely determined by the causality relation $\leq$. In case $\mathrm{E}$ is furthermore singular and cycle-free, every finite $X \in$ $L(\mathrm{E})$ can be seen to be in $S(\mathrm{E})$. We obtain

Proposition 2.3 Let E be a singular, conjunctive, cycle-free event structure with finite conflict. Then

$$
X \in L(\mathrm{E}) \Leftrightarrow\left\{\begin{array}{l}
\forall Y \subseteq_{f i n} X . \operatorname{Con}(Y) \wedge \\
\forall d, e \in E . d \leq e \in X \Rightarrow d \in X
\end{array}\right.
$$

$$
\text { and } \quad X \in S(\mathrm{E}) \Leftrightarrow X \in L(\mathrm{E}) \wedge X \text { is finite. }
$$

If $\mathrm{E}$ moreover is rooted and with binary conflict, then

$$
X \in L(\mathrm{E}) \Leftrightarrow\left\{\begin{array}{l}
\forall d, e \in X . \neg(d \# e) \wedge \\
\forall d, e \in E . d \leq e \in X \Rightarrow d \in X .
\end{array}\right.
$$

It follows that such structures can alternatively be represented as triples $\langle E$, Con,$\leq\rangle$ with Con $\subseteq \mathcal{P}_{\text {fin }}(E)$ and $\leq \subseteq E \times E$, as are the prime event structures of [23], respectively as triples $\langle E, \#, \leq\rangle$, as are the prime event structures of $[17,24]$.

\subsection{Infinitary secured configurations}

Neither the secured nor the left-closed configurations correspond exactly to the configurations used in Winskel [23, 24] to describe the behaviour of event structure. A generalisation of Winskel's notion of a configuration to the event structures of this paper is the following.

Definition 2.3 Let $\mathrm{E}=\langle E, \vdash\rangle$ be an event structure. The set $S^{\infty}(\mathrm{E})$ of infinitary secured configurations of $\mathrm{E}$ is given by

$$
X \in S^{\infty}(\mathrm{E}) \Leftrightarrow\left\{\begin{array}{l}
\forall n \in \mathbb{N} . \exists X_{n} \subseteq E . X=\bigcup_{n=0}^{\infty} X_{n} \\
\wedge X_{0}=\emptyset \wedge \forall n \in \mathbb{N} . X_{n} \subseteq X_{n+1} \wedge \\
\forall Y \subseteq X_{n+1} . \exists Z \subseteq X_{n} . Z \vdash Y .
\end{array}\right.
$$

Such a sequence $X_{0}, X_{1}, \ldots$ is called a stepwise securing of $X$. The infinitary secured configuration structure associated to $\mathrm{E}$ is $\mathcal{S}^{\infty}(\mathrm{E})=\left\langle E, S^{\infty}(\mathrm{E})\right\rangle$.

Computationally this notion can be understood by partitioning time in countably many successive intervals $I_{n}(n \geq 1)$. The set $X_{n}-X_{n-1}$ contains the events that occur in the interval $I_{n}$. These events must be enabled by events occurring in earlier intervals. The set $X$ contains all events that happen during such a run.

The secured configurations of Definition 1.5 can be explained by imposing two restrictions on the configurations above. First of all $X=X_{n}$ for some $n \in \mathbb{N}$, i.e., $X$ represents a state that can be reached in a finite amount of time; secondly $\left|X_{k}-X_{k-1}\right| \leq 1$ for $k=1, \ldots, n$, i.e., in each interval at most one event takes place. The following propositions connect these two notions.

Proposition 2.4 Let $\mathrm{E}=\langle E, \vdash\rangle$ be an event structure. Then $S(\mathrm{E})=\left\{X \in S^{\infty}(\mathrm{E}) \mid X\right.$ is finite $\}$.

Proof: Let $X$ be in $S(\mathrm{E})$. Take a securing $e_{1}, \ldots, e_{n}$ as exists by Definition 1.5, and let $X_{i}=\left\{e_{1}, \ldots, e_{i}\right\}$ for $i=0, \ldots, n$. Let $X_{i}=X_{n}$ for $i>n$. Then $X_{0}, X_{1}, \ldots$ is a stepwise securing of $X$.

Now let $X_{0}, X_{1}, \ldots$ be a stepwise securing of $X$, with $X$ finite. Then $X=X_{n}$ for some $n \in \mathbb{N}$. Furthermore, if $X_{k-1} \subseteq Y \subseteq X_{k}$ for some $1 \leq k \leq n$, then adding $Y$ between $X_{k-1}$ and $X_{k}$ preserves the property of the sequence being a stepwise securing. In this way the stepwise securing $X_{0}, \ldots, X_{n}$ can be refined into a stepwise securing $Y_{0}, \ldots, Y_{m}$ with $\left|Y_{k}-Y_{k-1}\right| \leq 1$ for $k=1, \ldots, m$. The latter can be written as a securing in the sense of Definition 1.5.

The proof above shows that events cannot be "synchronised" in event structures. If a finite number of events takes place simultaneously, they could just as well have occurred one after the other, in any order. 
The next proposition says that for certain event structures, including the ones from [17, 23, 24], the infinite secured configurations are completely determined by the finite ones.

Proposition 2.5 Let $\mathrm{E}=\langle E, \vdash\rangle$ be a singular event structure with finite causes and finite conflict. Then

$$
X \in S^{\infty}(\mathrm{E}) \Leftrightarrow \forall Y \subseteq \text { fin } X . \exists Z \in S(\mathrm{E}) . Y \subseteq Z \subseteq X,
$$

i.e. $\mathcal{S}^{\infty}(\mathrm{E})$ is the set of directed unions over $\mathcal{S}(\mathrm{E})$.

Proof: " $\Rightarrow "$ : Let $X \in S^{\infty}(\mathrm{E})$ and $Y \subseteq_{\text {fin }} X$. Let $X_{0}, X_{1}, \ldots$ be a stepwise securing of $X$ and choose $n$ in $\mathbb{N}$ such that $Y \subseteq X_{n}$. For $k=n, n-1, n-2, \ldots, 0$ choose the finite subset $Y_{k}$ of $X_{k}$ recursively as follows. $Y_{n}=Y$. Given $Y_{k}$ with $1 \leq k \leq n$, choose for any event $e \in Y_{k}$ a set $Z_{e} \subseteq X_{k-1}$ with $Z_{e} \vdash e$, and let $Y_{k-1}=\bigcup_{e \in Y_{k}} Z_{e}$. Because $\mathrm{E}$ has finite causes, the sets $Z_{e}$ are finite, and so is $Y_{k-1}$. As $\mathrm{E}$ is singular we have $\emptyset \vdash Z$ for any $Z \subseteq X$ with $|Z| \neq 1$. Therefore the sets $\bigcup_{i=0}^{k} Y_{i}$ for $k \leq n$ form a stepwise securing of the finite set $Z=\bigcup_{i=0}^{n} Y_{i}$. Hence, by Proposition 2.4, $Z \in S(\mathrm{E})$. Furthermore we have $Y \subseteq Z \subseteq X$.

" $\Leftarrow$ ": Let $X \subseteq E$ be such that the right-hand side holds. Take $X_{n}=\left\{e_{n} \mid \exists e_{1}, \ldots, e_{n-1} \in X\right.$. $\left.\forall k \leq n . \forall Y \subseteq\left\{e_{1}, \ldots, e_{k}\right\} . \exists Z \subseteq\left\{e_{1}, \ldots, e_{k-1}\right\} . Z \vdash Y\right\}$ for $n>0$, and take $X_{0}=\emptyset$. As a sequence $e_{1}, \ldots, e_{n}$ as occurs above can be prolonged by repeating events, we have $X_{n} \subseteq X_{n+1}$ for all $n \in \mathbb{N}$. By Definition 1.5 we have $X=\bigcup_{n=0}^{\infty} X_{n}$. Now let $Y \subseteq X_{n+1}$. It remains to be shown that $\exists Z \subseteq X_{n}$. $Z \vdash Y$. First suppose $Y=\emptyset$. As $S(\mathrm{E}) \neq \emptyset$, E must be rooted, and we may take $Z=\emptyset$. Now suppose $|Y|=1$. Then $\exists e_{1}, \ldots, e_{n+1} \in X . Y=\left\{e_{n+1}\right\} \wedge \forall k \leq n+1 . \forall Y^{\prime} \subseteq$ $\left\{e_{1}, \ldots, e_{k}\right\} . \exists Z \subseteq\left\{e_{1}, \ldots, e_{k-1}\right\} . Z \vdash Y^{\prime}$. So in particular $\exists Z \subseteq\left\{e_{1}, \ldots, e_{n}\right\} . Z \vdash Y$. Furthermore $Z \subseteq \bigcup_{i=1}^{n} X_{i}=X_{n}$. Finally suppose $|Y| \geq 2$. In case $Y$ is infinite we have $\emptyset \vdash Y$ since $\mathrm{E}$ is singular and with finite conflict. Otherwise there must be a $W \in S(\mathrm{E}) \subseteq L(\mathrm{E})$ with $Y \subseteq W \subseteq X$. Hence $\exists Z \subseteq W . Z \vdash Y$. As $\mathrm{E}$ is singular, $Z=\emptyset$.

Thus for singular event structures with finite causes and finite conflict we have $\mathcal{S}^{\infty}(\mathrm{E})=\mathcal{S}^{\infty}\left(\mathrm{E}^{\prime}\right)$ iff $\mathcal{S}(\mathrm{E})=\mathcal{S}\left(\mathrm{E}^{\prime}\right)$, i.e., $\mathcal{S}^{\infty}$-equivalence coincides with $\mathcal{S}$ equivalence.

Next, we augment Propositions 2.1 to 2.3 with characterisations of the infinitely secured configurations. To this end, we show that for the event structures of Proposition 2.1 their family of secured configurations is closed under consistent unions.

Lemma 1 Let E be a singular event structure with finite causes and finite conflict. Then

$$
X, Y \in S(\mathrm{E}) \wedge \operatorname{Con}(X \cup Y) \Rightarrow X \cup Y \in S(\mathrm{E}) .
$$

Proof: Let $e_{1}, \ldots, e_{n}$ be a securing of $X$ and $d_{1}, \ldots, d_{m}$ be one of $Y$, then $e_{1}, \ldots, e_{n}, d_{1}, \ldots, d_{m}$ is a securing of $X \cup Y$. The obtained securing may have repeated elements; if desired these can be eliminated by omitting all but the first occurrences of events.

Proposition 2.6 Let E be a singular event structure with finite causes and finite conflict. Then

$$
X \in S^{\infty}(\mathrm{E}) \Leftrightarrow\left\{\begin{array}{l}
\forall Y \subseteq_{\text {fin }} X . \operatorname{Con}(Y) \wedge \\
\forall e \in X . \exists e_{0}, \ldots, e_{n} \in X . e=e_{n} \wedge \\
\forall k \leq n .\left\{e_{0}, \ldots, e_{k-1}\right\} \vdash^{\prime} e_{k} .
\end{array}\right.
$$

Proof: " $\Rightarrow$ " follows immediately from Propositions 2.5 and 2.1. For " $\Leftarrow$ " let $X \subseteq E$ be such that the righthand side holds. Let $Y \subseteq \subseteq_{\text {fin }} X$. Using Proposition 2.5 it suffices to find a $Z \in S(\mathrm{E})$ with $Y \subseteq Z \subseteq X$. For any $e \in Y$ let $Z_{e}=\left\{e_{0}, \ldots, e_{n}\right\}$ with $e_{0}, \ldots, e_{n}$ a sequence as mentioned above. Then $Z_{e} \in S(\mathrm{E})$ by Proposition 2.1 and so $Z=\bigcup_{e \in Y} Z_{e} \in S(\mathrm{E})$ by Lemma 1 . Moreover, $Y \subseteq Z \subseteq X$.

In case $\mathrm{E}$ has binary conflict we even have

$$
X \in S^{\infty}(\mathrm{E}) \Leftrightarrow\left\{\begin{array}{l}
\forall d, e \in X . \neg(d \# e) \wedge \\
\forall e \in X . \exists e_{0}, \ldots, e_{n} \in X . e=e_{n} \wedge \\
\forall k \leq n .\left\{e_{0}, \ldots, e_{k-1}\right\} \vdash^{\prime} e_{k} .
\end{array}\right.
$$

Proposition 2.7 Let E be a singular, conjunctive, $\mathcal{S}$ irredundant event structure with finite causes and finite conflict. Then $S^{\infty}(\mathrm{E})=L(\mathrm{E})$.

Proof: " $\subseteq$ " follows immediately from Definition 2.3. " $\supseteq$ ": For any $e \in E$, let $\downarrow e$ be $\{d \in E \mid d \leq e\}$. Any secured or left-closed configuration containing $e$ must contain $\downarrow e$. As $\mathrm{E}$ is $\mathcal{S}$-irredundant, $e$ must occur in a secured configuration. Hence $\downarrow e$ is finite, and Con $(\downarrow e)$. Thus $\downarrow e \in S(\mathrm{E})$ by Proposition 2.3.

Now suppose $X \in L(\mathrm{E})$. For any $Y \subseteq_{f i n} X$, let $\downarrow Y$ be $\bigcup_{e \in Y} \downarrow e$. It must be that $\downarrow Y \subseteq_{\text {fin }} X$. Hence $\operatorname{Con}(\downarrow Y)$. Now Lemma 1 implies that $\downarrow Y \in S(\mathrm{E})$. Moreover, $Y \subseteq \downarrow Y \subseteq X$, so Proposition 2.5 implies that $X \in S^{\infty}(\mathrm{E})$.

\subsection{The event structures of WINSKEL [23]}

These are defined as triples $\mathrm{E}=\langle E, C o n, \vdash\rangle$ where

- $E$ is a set of events,

- $C o n \subseteq \mathcal{P}_{f i n}(E)$ is a nonempty consistency predicate such that: $Y \subseteq X \in C o n \Rightarrow Y \in C o n$,

- and $\vdash \subseteq$ Con $\times E$ is the enabling relation, which satisfies $X \vdash e \wedge X \subseteq Y \in C o n \Rightarrow Y \vdash e$.

Such an event structure is stable if it satisfies

$$
X \vdash e \wedge Y \vdash e \wedge \operatorname{Con}(X \cup Y \cup\{e\}) \Rightarrow X \cap Y \vdash e .
$$


The family $S^{\infty}$ (E) of configurations of such an event structure (written $\mathcal{F}(\mathrm{E})$ in [23]) consists of those $X \subseteq$ $E$ which are

- consistent: every finite subset of $X$ is in Con,

- and secured: $\forall e \in X . \exists e_{0}, \ldots, e_{n} \in X . e_{n}=e \wedge$ $\forall i \leq n .\left\{e_{0}, \ldots, e_{i-1}\right\} \vdash e_{i}$,

just as in Proposition 2.6. In addition, $L(\mathrm{E})$ and $S(\mathrm{E})$ can be defined exactly as in Proposition 2.1 (reading $\vdash$ for $\left.\vdash^{\prime}\right)$. Again, we write $\mathcal{L}(\mathrm{E})$ for $\langle E, L(\mathrm{E})\rangle$, and likewise for $\mathcal{S}(\mathrm{E})$ and $\mathcal{S}^{\infty}(\mathrm{E})$.

Here we will show that up to $\mathcal{L}$-, $\mathcal{S}$ - and $\mathcal{S}^{\infty}$ equivalence these event structures are exactly the ones in our sense which are rooted, singular, with finite causes and with finite conflict; and the stable event strutures of [23] are the ones which are moreover locally conjunctive.

For $\mathrm{E}=\left\langle E, C o n, \vdash^{\prime}\right\rangle$ an event structure as in [23], let the event structure $\mathcal{E}(\mathrm{E})=\langle E, \vdash\rangle$ be given by

$$
X \vdash Y \text { iff }\left\{\begin{array}{l}
\text { either } Y=\{e\}, \operatorname{Con}(\{e\}) \text { and } X \vdash^{\prime} e \\
\text { or }|Y| \neq 1, X=\emptyset \text { and } \operatorname{Con}(Y) \\
\text { or } Y \text { is infinite and } X=\emptyset .
\end{array}\right.
$$

Proposition 2.8 Let $\mathrm{E}$ be an event structure as in [23]. Then $\mathcal{E}(\mathrm{E})$ is rooted, singular and with finite causes and finite conflict. If $\mathrm{E}$ is stable then $\mathcal{E}(\mathrm{E})$ is locally conjunctive. Moreover, $\mathcal{L}(\mathcal{E}(\mathrm{E}))=\mathcal{L}(\mathrm{E})$, $\mathcal{S}(\mathcal{E}(\mathrm{E}))=\mathcal{S}(\mathrm{E})$ and $S^{\infty}(\mathcal{E}(\mathrm{E}))=S^{\infty}(\mathrm{E})$.

Proof: Let $\mathrm{E}=\left\langle E, C o n, \vdash^{\prime}\right\rangle$ be an event structure as in [23]. As Con is nonempty and subset-closed we have $\emptyset \in$ Con. Thus $\emptyset \vdash \emptyset$, i.e. $\mathcal{E}(\mathrm{E})$ is rooted. By construction, $\mathcal{E}(\mathrm{E})$ is singular and with finite causes and finite conflict. That the stability of $\mathrm{E}$ implies the local conjunctivity of $\mathcal{E}(\mathrm{E})$ follows because every collection of finite sets has a finite subcollection with the same intersection. With Propositions 2.1 and 2.6 one easily checks that $\mathcal{L}(\mathcal{E}(\mathrm{E}))=\mathcal{L}(\mathrm{E}), \mathcal{S}(\mathcal{E}(\mathrm{E}))=\mathcal{S}(\mathrm{E})$ and $S^{\infty}(\mathcal{E}(\mathrm{E}))=S^{\infty}(\mathrm{E})$.

For $\mathrm{E}=\langle E, \vdash\rangle$ a rooted event structure, the structure $\mathcal{W}(\mathrm{E})=\left\langle E, C o n, \vdash^{\prime}\right\rangle$, where $C o n$ and $\vdash^{\prime}$ are given by Definition 2.1, is clearly an event structure in the sense of [23].

Proposition 2.9 Let E be a rooted, singular event structure with finite causes and finite conflict. Then $\mathcal{L}(\mathcal{W}(\mathrm{E}))=\mathcal{L}(\mathrm{E}), \mathcal{S}(\mathcal{W}(\mathrm{E}))=\mathcal{S}(\mathrm{E})$ and $S^{\infty}(\mathcal{W}(\mathrm{E}))=$ $S^{\infty}(\mathrm{E})$. Moreover, $\mathcal{W}(\mathrm{E})$ is stable if $\mathrm{E}$ is locally conjunctive.

Proof: Trivial, with Propositions 2.1 and 2.6

\subsection{The event structures of WINSKEL}

These are defined as triples $\mathrm{E}=\langle E, \#, \vdash\rangle$ where

- $E$ is a set of events,

- \# $\subseteq E \times E$ is a symmetric, irreflexive conflict relation. Write $C o n$ for the set of finite, conflictfree subsets of $E$, i.e. those finite subsets $X \subseteq E$ for which

$$
\forall e, e^{\prime} \in X . \neg\left(e \# e^{\prime}\right),
$$

- and $\vdash \subseteq C$ Con $\times E$ is the enabling relation, which satisfies $X \vdash e \wedge X \subseteq Y \in C o n \Rightarrow Y \vdash e$.

Such an event structure is stable if it satisfies

$$
X \vdash e \wedge Y \vdash e \wedge \operatorname{Con}(X \cup Y \cup\{e\}) \Rightarrow X \cap Y \vdash e .
$$

The family $S^{\infty}(\mathrm{E})$ of configurations of such an event structure (written $\mathcal{F}(\mathrm{E})$ in [24]) consists of those $X \subseteq$ $E$ which are

- conflict-free: $\forall e, e^{\prime} \in X . \neg\left(e \# e^{\prime}\right)$,

- and secured: $\forall e \in X . \exists e_{0}, \ldots, e_{n} \in X . e_{n}=e \wedge$ $\forall i \leq n .\left\{e_{0}, \ldots, e_{i-1}\right\} \vdash e_{i}$.

Note that a set of events $X$ is conflict-free iff every finite subset of $X$ is in $C o n$. In addition, $L(\mathrm{E})$ and $S(\mathrm{E})$ can be defined exactly as in Proposition 2.2 (reading $\vdash$ for $\vdash^{\prime}$ ).

Say that an event structure $\langle E, C o n, \vdash\rangle$ in the sense of [23] has binary conflict if for any $X \subseteq_{\text {fin }} E$ :

$$
\operatorname{Con}(X) \Leftrightarrow \forall Y \subseteq X .(|Y|=2 \Rightarrow \operatorname{Con}(Y)) .
$$

Clearly, the event structures of [24] are just a reformulation of the event structures of [23] that have binary conflict. A small variation of the arguments from the previous section shows that, up to $\mathcal{L}-, \mathcal{S}_{-}$and $\mathcal{S}^{\infty}$ equivalence, the event structures of [24] are exactly the ones in our sense which are rooted, singular, with finite causes and with binary conflict; and the stable event strutures of [24] are the ones which are moreover locally conjunctive.

\subsection{The prime event structures of [23]}

These are defined as triples $\mathrm{E}=\langle E, C o n, \leq\rangle$ where

- $E$ is a set of events,

- $C o n \subseteq \mathcal{P}_{\text {fin }}(E)$ is a nonempty consistency predicate such that: $Y \subseteq X \in C o n \Rightarrow Y \in C o n$, and $\{e\} \in C o n$ for all $e \in E$,

- and $\leq \subseteq E \times E$ is a partial order, the causality relation, satisfying

$-d \leq e \in X \in$ Con $\Rightarrow X \cup\{d\} \in$ Con

- and $\downarrow e=\{d \in E \mid d \leq e\}$ is finite for all $e \in E$. 
The set $L(\mathrm{E})$ of configurations of such an event structure consists of those $X \subseteq E$ which are

- consistent: every finite subset of $X$ is in Con,

- and left-closed: $\forall d, e \in E . d \leq e \in X \Rightarrow d \in X$,

just as in Proposition 2.3. In addition, $S(\mathrm{E})$ could be defined as the set of finite configurations in $L(\mathrm{E})$.

Here we will show that up to $\mathcal{L}$ and $\mathcal{S}$-equivalence these event structures are exactly the ones in our sense which are rooted, singular, conjunctive, $\mathcal{S}$-irredundant and with (finite causes and) finite conflict.

For $\mathrm{E}=\langle E$, Con,$\leq\rangle$ a prime event structure as in [23], let the event structure $\mathcal{E}(\mathrm{E})=\langle E, \vdash\rangle$ be given by

$$
X \vdash Y \text { iff }\left\{\begin{array}{l}
Y=\{e\} \text { and } X=\{d \mid d<e\} \\
\text { or }|Y| \neq 1, X=\emptyset \text { and } \operatorname{Con}(Y) \\
\text { or } Y \text { is infinite and } X=\emptyset .
\end{array}\right.
$$

Proposition 2.10 Let E be a prime event structure as in [23]. Then $\mathcal{E}(\mathrm{E})$ is rooted, singular, conjunctive, $\mathcal{S}$-irredundant and with finite causes and finite conflict. Moreover, $\mathcal{L}(\mathcal{E}(\mathrm{E}))=\mathcal{L}(\mathrm{E})$ and $\mathcal{S}(\mathcal{E}(\mathrm{E}))=\mathcal{S}(\mathrm{E})$.

Proof: Let $\mathrm{E}=\langle E$, Con,$\leq\rangle$ be a prime event structure as in [23]. As Con is nonempty and subset-closed we have $\emptyset \in C$ Con. Thus $\emptyset \vdash \emptyset$, i.e. $\mathcal{E}(\mathrm{E})$ is rooted. By construction, $\mathcal{E}(\mathrm{E})$ is singular and with finite causes and finite conflict. As for every set of events $Y$ there is at most one set of events $X$ with $X \vdash Y, \mathcal{E}(\mathrm{E})$ is surely conjunctive. With Proposition 2.3 one easily checks that $L(\mathcal{E}(\mathrm{E}))=L(\mathrm{E})$, and hence $\mathcal{L}(\mathcal{E}(\mathrm{E}))=\mathcal{L}(\mathrm{E})$ and $\mathcal{S}(\mathcal{E}(\mathrm{E}))=\mathcal{S}(\mathrm{E})$. For every event $e \in E$ we have $e \in \downarrow e \in S(\mathrm{E})=S(\mathcal{E}(\mathrm{E}))$. Hence $\mathcal{E}(\mathrm{E})$ is $\mathcal{S}$ irredundant.

For $\mathrm{E}=\langle E, \vdash\rangle$ a rooted, $\mathcal{S}$-irredundant event structure, the structure $\mathcal{W}^{\prime}(\mathrm{E})=\left\langle E, C_{o n}{ }^{\prime}, \leq\right\rangle$, where $\operatorname{Con}^{\prime}(X)$ iff $\operatorname{Con}(\{d \in E \mid \exists e \in X . d \leq e\})$, and Con and $\leq$ are given by Definition 2.1, is clearly a prime event structure in the sense of [23]. In particular, for all $e \in E$, the set $\downarrow e$ has to be finite, and $\operatorname{Con}(\{e\})$, since otherwise $e$ could not occur in any secured configuration, contradicting $\mathcal{S}$-irredundancy. As $\mathcal{S}$-irredundancy implies cycle-freeness, $\leq$ must be a partial order.

Proposition 2.11 Let E be a rooted, singular, conjunctive, $\mathcal{S}$-irredundant event structure with finite conflict. Then $\mathcal{L}\left(\mathcal{W}^{\prime}(\mathrm{E})\right)=\mathcal{L}(\mathrm{E})$ and $S\left(\mathcal{W}^{\prime}(\mathrm{E})\right)=S(\mathrm{E})$.

Proof: Trivial, with Proposition 2.3.

Note that the requirement of having finite causes is not needed in Proposition 2.11. It follows that, preserving
$\mathcal{L}$ - and $\mathcal{S}$-equivalance, any rooted, singular, conjunctive, $\mathcal{S}$-irredundant event structure with finite conflict can be converted into one that has also finite causes.

On the class of event structures corresponding to the prime event structures of [23], Proposition 2.7 says that $S^{\infty}$ coincides with $L$. Thus each of $S^{\infty}$ and $L$ can be understood as generalisation of the notion of configuration for prime event structures from [23].

\subsection{The event structures of $[17]$}

These are triples $\mathrm{E}=\langle E, \leq, \#\rangle$ where

- $E$ is a set of events,

- $\leq \subseteq E \times E$ is a partial order, the causality relation,

- $\# \subseteq E \times E$ is an irreflexive, symmetric relation, the conflict relation, satisfying

$$
\forall d, e, f \in E . d \leq e \wedge d \# f \Rightarrow e \# f
$$

the principle of conflict heredity.

The set $L(\mathrm{E})$ of configurations of such an event structure consists of those $X \subseteq E$ which are

- conflict-free: \# $\#(X \times X)=\emptyset$,

- and left-closed: $\forall d, e \in E . d \leq e \in X \Rightarrow d \in X$,

just as in Proposition 2.3. In addition, $S(\mathrm{E})$ could be defined as the set of finite configurations in $L(\mathrm{E})$.

The prime event structures of [24] are defined likewise, but additionally requiring

$$
\{d \in E \mid d \leq e\} \text { is finite for all } e \in E,
$$

the principle of finite causes.

Here we will show that up to $\mathcal{L}$ and $\mathcal{S}$-equivalence these event structures are exactly the ones in our sense which are rooted, singular, conjunctive, $\mathcal{L}$ irredundant, cycle-free and with binary conflict, and for the ones from [24] also $\mathcal{S}$-irredundant.

For $\mathrm{E}=\langle E, \leq, \#\rangle$ a prime event structure as in [17], let the event structure $\mathcal{E}(\mathrm{E})=\langle E, \vdash\rangle$ be given by

$$
X \vdash Y \text { iff }\left\{\begin{array}{l}
Y=\{e\} \text { and } X=\{d \mid d<e\} \\
\text { or } Y=\{d, e\}, d \neq e, X=\emptyset \text { and } \neg(d \# e) \\
\text { or }|Y| \neq 1,2 \text { and } X=\emptyset .
\end{array}\right.
$$

Proposition 2.12 Let $\mathrm{E}$ be an event structure as in [17]. Then $\mathcal{E}(\mathrm{E})$ is rooted, singular, conjunctive, $\mathcal{L}$ irredundant, cycle-free and with binary conflict. If $\mathrm{E}$ satisfies the principle of finite causes then $\mathcal{E}(\mathrm{E})$ is moreover $\mathcal{S}$-irredundant. Furthermore, $\mathcal{L}(\mathcal{E}(\mathrm{E}))=\mathcal{L}(\mathrm{E})$ and $\mathcal{S}(\mathcal{E}(\mathrm{E}))=\mathcal{S}(\mathrm{E})$.

Proof: Let $\mathrm{E}=\langle E, \leq, \#\rangle$ be an event structure as in [17]. By construction, $\mathcal{E}(\mathrm{E})$ is rooted, singular and 
with binary conflict. As for every set of events $Y$ there is at most one set of events $X$ with $X \vdash Y, \mathcal{E}(\mathrm{E})$ is surely conjunctive. The relation $\prec$ coincides with $<$, so $\mathcal{E}(\mathrm{E})$ is cycle-free. With Proposition 2.3, trivially $L(\mathcal{E}(\mathrm{E}))=L(\mathrm{E})$. Hence $\mathcal{L}(\mathcal{E}(\mathrm{E}))=\mathcal{L}(\mathrm{E})$ and $\mathcal{S}(\mathcal{E}(\mathrm{E}))=\mathcal{S}(\mathrm{E})$.

For every $e \in E$, the set $\downarrow e=\{d \in E \mid d \leq e\}$ must be conflict-free, by the principle of conflict heredity and the irreflexivity of \#. Hence, $e \in \downarrow e \in L(\mathrm{E})=$ $L(\mathcal{E}(\mathrm{E}))$. Therefore $\mathcal{E}(\mathrm{E})$ is $\mathcal{L}$-irredundant. In case $\mathrm{E}$ satisfies the principle of finite causes, $\downarrow e$ is moreover finite and $e \in \downarrow e \in S(\mathrm{E})=S(\mathcal{E}(\mathrm{E}))$. In this case $\mathcal{E}(\mathrm{E})$ is even $\mathcal{S}$-irredundant.

For $\mathrm{E}=\langle E, \vdash\rangle$ a rooted, $\mathcal{L}$-irredundant, cycle-free event structure, the structure $\mathcal{W}_{N P}(\mathrm{E})=\left\langle E, \leq, \#^{\prime}\right\rangle$, where $d \#^{\prime} e$ iff $\exists d^{\prime} \leq d . \exists e^{\prime} \leq e \cdot d^{\prime} \# e^{\prime}$, and $\leq$ and $\#$ are given by Definition 2.1, is clearly an event structure in the sense of [17]. In particular, $\leq$ is a partial order since $\mathrm{E}$ is cycle-free, and $\#^{\prime}$ is irreflexive since if $e \#^{\prime} e$ then $e$ could not occur in any configuration, contradicting $\mathcal{L}$-irredundancy. In case $\mathrm{E}$ is moreover $\mathcal{S}$-irredundant, then the sets $\downarrow e$ have to be finite, since otherwise $e$ could not occur in any secured configuration. In this case $\mathcal{W}_{N P}(\mathrm{E})$ is a prime event structure as in [24].

Proposition 2.13 Let E be a rooted, singular, conjunctive, $\mathcal{L}$-irredundant and cycle-free event structure with binary conflict. Then $\mathcal{L}\left(\mathcal{W}_{N P}(\mathrm{E})\right)=\mathcal{L}(\mathrm{E})$ and $\mathcal{S}\left(\mathcal{W}_{N P}(\mathrm{E})\right)=\mathcal{S}(\mathrm{E})$.

Proof: Trivial, with Proposition 2.3.

If $\mathrm{E}$ falls in the class of event structures that according to Proposition 2.13 is $\mathcal{L}$-equivalent to a prime event structure in the sense of [24], then $\mathcal{S}^{\infty}(\mathrm{E})=\mathcal{L}(\mathrm{E})$, by Proposition 2.7. This does not extend to the structures corresponding to the event structures of [17] however:

Example 3 Let $E$ be given by $E=\left\{e_{0}, e_{1}, \ldots\right\} \cup\left\{e_{\infty}\right\}$, $\#=\emptyset$ and $e_{i}<e_{j}$ iff $i<j$. Then $E \in L(\mathrm{E})$ but $E \notin S^{\infty}(\mathrm{E})$.

\subsection{Summary and remarks}

The left-closed configurations of an event structure generalise the left-closed and conflict-free subsets of events considered in Nielsen, Plotkin \& Winskel [17], as well as the families of configurations of prime event structures as considered in WinskeL [23, 24]. The infinitary secured configurations generalise the families of configurations of event structures (prime and otherwise) considered in [23, 24]. The families of configurations of such event structures are completely determined by their finite secured configurations. Now up to $\mathcal{L}$ - and $\mathcal{S}^{\infty}$ - and $\mathcal{S}$-equivalence the following correspondences have been established.

\begin{tabular}{l|l|r|}
\hline ev. str. [23] & rtd, sing, f.causes \& f.conflict & $\mathcal{S}^{\infty}, \mathcal{L}$ \\
stable [23] & same \& locally conjunctive & $\mathcal{S}^{\infty}, \mathcal{L}$ \\
prime [23] & same \& conjunctive \& $\mathcal{S}$-irr. & $\mathcal{S}^{\infty}, \mathcal{L}$ \\
ev. str. [24] & rtd, sing, f.causes \& bin.conflict & $\mathcal{S}^{\infty}, \mathcal{L}$ \\
stable [24] & same \& locally conjunctive & $\mathcal{S}^{\infty}, \mathcal{L}$ \\
prime [24] & same \& conjunctive \& $\mathcal{S}$-irr. & $\mathcal{S}^{\infty}, \mathcal{L}$ \\
ev. str. [17] & rtd, sing, b.c., conj, $\mathcal{L}$-irr \& c.-f. & $\mathcal{S}, \mathcal{L}$ \\
\hline
\end{tabular}

In the characterisations of the prime event structures from [23] and [24] the requirement of having finite causes is optional, i.e. any rooted, singular, conjunctive, $\mathcal{S}$-irredundant event structure with finite conflict can be converted into one that also has finite causes.

Any event structure $\mathrm{E}=\langle E, \vdash\rangle$ can be converted into an $\mathcal{S}$-irredundant structure, namely by omitting from $E$ all events that do not occur in any secured configuration, and omitting from $\vdash$ any enablings $X \vdash Y$ in which such events occur in $X$ or $Y$. This clearly preserves $S(\mathrm{E})$, as well as the properties rootedness, singularity, conjunctivity and having finite causes and finite or binary conflict. If $\mathrm{E}$ is singular and with finite causes and finite conflict, it also preserves $S^{\infty}(\mathrm{E})$, by Proposition 2.5. Thus, up to having the same infinitary secured configurations, the prime event structures of [23] (resp. [24]) even correspond to the class of our event structures that are rooted, singular, conjunctive and with finite (resp. binary) conflict, i.e. not requiring $\mathcal{S}$-irredundancy. However, it should be noted that this correspondence does not hold up to $\mathcal{S}^{\infty}$-equivalence, as the set of events is not preserved.

Preserving $\mathcal{S}$-equivalence, any event structure can be converted into one with finite causes and finite conflict, namely by adding all enablings $\emptyset \vdash Y$ with $Y$ infinite, and omitting the enablings $X \vdash Y$ with $X$ infinite. This procedure preserves the other properties of Definition 2.2, except $\mathcal{L}$-irredundancy. Hence, up to $\mathcal{S}$-equivalence the correspondences hold without finite causes and finite conflict.

Finally, also preserving $\mathcal{S}$-equivalence, every event structure can be converted into a finitary one, namely by omitting all enablings of the form $X \vdash Y$ with $Y$ infinite. This conversion preserves the properties of Definition 2.2, except $\mathcal{L}$-irredundancy and, of course, finite conflict; it preserves binary conflict only in the form

$$
2<|X|<\infty \Rightarrow \emptyset \vdash X
$$




\section{Comparing Models}

Having seen the general correspondences between our various models of computation - event structures, configuration structures and propositional theories - we now trace the relationships for various natural subclasses; we are guided by the exploration of previous notions of event structures in the last section (Petri Nets are tied in in Section 3.3). In Section 3.1 we give properties of configuration structures corresponding to those of event structures, and provide corresponding classes of prepositional theories (described according to the syntactic form of the allowed formulae). In Section 3.2 we tackle the converse completeness problem: given a collection of properties of a configuration structure is there an event structure (or a propositional theory) satisfying the corresponding properties and which yields the given configuration structure? Note that, following our general point of view, we understand the configuration structures as providing our (semantic) model of behaviour, and so we use them to mediate the comparisons between the (more syntactic) models of computation.

\section{1 corresponding behaviours}

\begin{tabular}{|l|l|l|}
\hline $\begin{array}{l}\text { Event } \\
\text { structures }\end{array}$ & $\begin{array}{l}\text { Configuration } \\
\text { structures }\end{array}$ & $\begin{array}{l}\text { Propositional } \\
\text { theories }\end{array}$ \\
\hline \hline pure & - & pure \\
rooted & rooted & $(\geq 0$, any $)$ \\
finitary & finitary & finitary \\
singular & closed under $\bar{U}$ & $(1$, any), (any, 0) \\
conjunctive & closed under $\bigcap_{\bullet}$ & (any, $\leq 1)$ \\
locally conj. & closed under $\bar{\bigcap}$ & (any, ddc) \\
finite confl. & finite conflict & (finite, any) \\
binary confl. & binary conflict & $(\leq 2$, any $)$ \\
\hline
\end{tabular}

TABLE 1: Corresponding properties

Table 1 gives the various corresponding properties. We have already defined all those needed for event structures. For configuration structures $\bigcap_{\bullet}, \bar{\bigcap}_{\bullet}$ and $\bar{U}$ stand for closure under nonempty intersections, nonempty bounded intersections and bounded unions respectively, where these notions and two others - of finite and binary conflict - are defined as follows:

Definition 3.1 Let $\mathrm{C}=\langle E, C\rangle$ be a configuration structure. A set of events $X \subseteq E$ is consistent, written $\operatorname{Con}(X)$, if $\exists Y \in C . X \subseteq Y$. C is said to be closed under bounded unions $(\bar{\bigcup})$ if

$$
X_{i} \in C(\text { for } i \in I) \wedge C \operatorname{Con}\left(\bigcup_{i \in I} X_{i}\right) \Rightarrow \bigcup_{i \in I} X_{i} \in C .
$$

$\mathrm{C}$ is closed under nonempty intersections $\left(\bigcap_{\bullet}\right)$ if

$$
X_{i} \in C(\text { for } i \in I \neq \emptyset) \Rightarrow \bigcap_{i \in I} X_{i} \in C
$$

and under bounded nonempty intersections $\left(\bar{\bigcap}_{\bullet}\right)$ if

$X_{i} \in C($ for $i \in I \neq \emptyset) \wedge \operatorname{Con}\left(\bigcup_{i \in I} X_{i}\right) \Rightarrow \bigcap_{i \in I} X_{i} \in C$.

$\mathrm{C}$ has finite conflict if

$[\forall Y \subseteq X .(Y$ finite $\Rightarrow \exists Z \in C . Y \subseteq Z \subseteq X)] \Rightarrow X \in C$

and binary conflict if

$[\forall Y \subseteq X .(|Y| \leq 2 \Rightarrow \exists Z \in C . Y \subseteq Z \subseteq X)] \Rightarrow X \in C$.

The first three conditions are particularly natural as they are couched in terms of the order-theoretic structure of sets of events, and do not involve discussion of individual events. A natural replacement of this kind for finite conflict is closure under directed unions. This property is in fact strictly weaker than finite conflict as the configuration structure consisting of the co-singleton sets of natural numbers illustrates. However the two properties coincide for those configuration structures closed under non-empty intersections.

A configuration-oriented condition corresponding to binary conflict is coherence:

Definition 3.2 Let $\mathrm{C}=\langle E, C\rangle$ be a configuration structure. It is coherent iff for every nonempty family $X_{i}(i \in I)$ of configurations:

$\left[\forall j, k \exists Y \in C . X_{j} \cap X_{j} \subseteq U \subseteq Y \subseteq \cap_{i \in I} X_{i}\right] \Rightarrow \cap_{i \in I} X_{i} \in C$.

As before, binary conflict is the stronger, with the cosingletons again providing a counterexample; and, as before, in the presence of closure under non-empty intersections, the two conditions coincide.

Proposition 3.1 Let $\mathrm{E}$ be an event structure. If $\mathrm{E}$ has any of the properties of Table 1 , then $\mathcal{L}(\mathrm{E})$ has the corresponding property.

Proof: The details are routine and are omitted.

When dealing with combinations of properties a little more information will be helpful.

Definition 3.3 Let $\mathrm{C}=\langle E, C\rangle$ be a configuration structure. It has strong finite conflict iff

$\forall Y \subseteq X .(|Y|=1 \Rightarrow \exists Z \in C . Y \subseteq Z \subseteq X) \wedge(Y$ finite $\Rightarrow C o n(Y)]$ and it has strong binary conflict iff

$\forall Y \subseteq X .(|Y|=1 \Rightarrow \exists Z \in C . Y \subseteq Z \subseteq X) \wedge(|Y| \leq 2 \Rightarrow \operatorname{Con}(Y$ 
Proposition 3.2 (continued) Let E be a singular event structure. If it has finite conflict then $\mathcal{L}(\mathrm{E})$ has strong finite conflict, and if it has binary conflict then $\mathcal{L}(\mathrm{E})$ has strong binary conflict.

We now turn to axiomatisations. We say that a formula has the form (any, $\leq 1$ ) if it is a clause with no restriction on its antecedents and with at most one consequent; and and (any, ddc) indicates an implication whose premise can be any conjunction of propositional letters, and whose conclusion has the "ddc" form. This is a formula $\bigvee_{j \in J} \wedge Z_{j}$ where the $Z_{j}$ are sets of letters, and we write $\vee \Phi$ for $(\bigvee \Phi) \wedge \bigwedge\left\{\neg\left(\phi \wedge \phi^{\prime}\right) \mid \phi, \phi^{\prime} \in \Phi, \phi \neq \phi^{\prime}\right\}$, the disjoint disjunction of $\Phi$. In case all the $Z_{j}$ are finite, we have the "ddfc" form. The meaning of the other forms of formula used in Table 1 should now be self-evident.

Proposition 3.3 Let $T$ be a set of formulae over a set $E$ all of whose elements have one of the forms given in a row of Table 1 . Then $M(T)$ has the corresponding property, as given in the table.

Proof: We consider only the first case, leaving the others to the reader To this end we show that the collection of models of a family of implications of the form $\bigwedge X \Rightarrow \bigvee_{j \in J} \bigwedge Z_{j}$ is closed under bounded nonempty intersections. Suppose that $\left\{m_{i} \mid i \in I\right\}$ is such a collection, with upper bound $m^{\prime}$. Let $m$ be the intersection of the $m_{i}$; we must show it is a model. To this end, choose one implication of the above form and suppose that $m$ includes its premise $X$. Then so does $m^{\prime}$ and hence there is a unique $j$ in $J$ such that $Z_{j} \subseteq m^{\prime}$; but since, for any $i$ in $I, m_{i}$ is a model with $X \subseteq m_{i} \subseteq m^{\prime}$ we have that $Z_{j} \subseteq m_{i}$. So $Z_{j} \subseteq m$, and this must be the unique such $j$ as $m \subseteq m^{\prime}$, since $I$ is non-empty.

\subsection{Completeness}

We begin with the relationship between event structures and configuration structures.

Theorem 3 A configuration structure $\mathrm{C}$ has all of the properties of a given row of Table ?? iff there is a (pure) event structure E with the corresponding properties such that $\mathcal{L}(\mathrm{E})=\mathrm{C}$. The same holds with the addition of rootedness to all the entries.

Proof: The implications from right to left follow at once from Proposition ??.

For the converse, let $\mathrm{C}=(E, C)$ be a configuration structure. We first consider $\mathrm{E}=E(\mathrm{C})$; by Proposition ?? we have that $\mathcal{L}(\mathrm{E})=\mathrm{C}$. It is straightforward to check that
- $\mathrm{E}$ is always pure,

- if $\mathrm{C}$ is rooted, then so is $\mathrm{E}$,

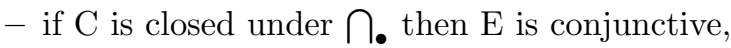

- and if $\mathrm{C}$ is $\bar{\bigcap}_{\text {- }}$-closed then $\mathrm{E}$ is locally conjunctive.

This construction therefore covers the cases of rows 1 and 5 of the table, with or without rootedness.

Next let $\mathrm{C}$ have finite conflict. Set $\mathrm{E}=\left(E, \vdash \cup \vdash^{\omega}\right)$ with $\vdash$ defined as before, and $X \vdash^{\omega} Y$ iff $X=\emptyset$ and $Y$ infinite. It is straightforward to check that

- E is always pure and with finite conflict,

- if $\mathrm{C}$ is rooted, then so is $\mathrm{E}$,

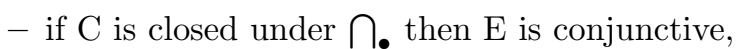

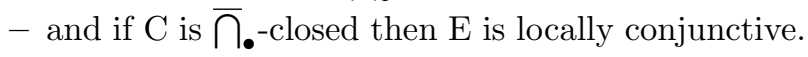

We show that $\mathcal{L}(\mathrm{E})=\mathrm{C}$. That $C \subseteq L(\mathrm{E})$ goes exactly as in the previous case, so suppose $X \in \mathcal{L}(\mathrm{E})$. For any finite $Y \subseteq X$ there must be a $Z \subseteq X$ with $Z \vdash Y$. By construction, $Z \cup Y \in C$. As $Y \subseteq Z \cup Y \subseteq X$, and $\mathrm{C}$ has finite conflict, we have $X \in C$.

This construction therefore covers the cases of rows 2, 6 and 13 of the table, with or without rootedness. The case where $\mathrm{C}$ has binary conflict goes similarly.

Now assume $\mathrm{C}$ is closed under bounded unions $(\overline{\mathrm{U}})$. Let $\mathrm{E}=\left(E, \vdash_{1} \cup \vdash_{2}\right)$ with

$$
\begin{aligned}
& X \vdash_{1} Y \text { iff }|Y|=1, X \cap Y=\emptyset \text { and } X \cup Y \in C, \\
& X \vdash_{2} Y \text { iff } X=\emptyset,|Y| \neq 1 \text { and } C o n(Y) .
\end{aligned}
$$

It is straightforward to check that

- E is always pure and singular,

- if $\mathrm{C}$ is rooted, then so is $\mathrm{E}$,

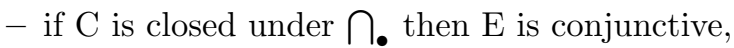

- and if $\mathrm{C}$ is $\bar{\bigcap}_{\text {. }}$-closed then $\mathrm{E}$ is locally conjunctive.

We show that $\mathcal{L}(\mathrm{E})=\mathrm{C}$. Suppose $X \in C$. For any $Y \subseteq X$ take $Z=X-Y$ if $|Y|=1$ and $Z=\emptyset$ otherwise. Then $Z \subseteq X$ and $Z \vdash Y$. So $X \in \mathcal{L}(\mathrm{E})$. Conversely, suppose $X \in \mathcal{L}(\mathrm{E})$. Then there is a $Z \subseteq X$ such that $Z \vdash X$. In case $|X|=1$ we have $X=Z \cup X \in C$. In case $|X|>1$ it must be that $Z=\emptyset$ and $\operatorname{Con}(X)$. Moreover, for any $e \in X$ there is a $Z_{e} \subseteq X$ such that $Z_{e} \vdash\{e\}$. By construction, $Z_{e} \cup\{e\} \in C$. As $\bigcup_{e \in X}\left(Z_{e} \cup\{e\}\right)=X$ and $\operatorname{Con}(X)$, and $\mathrm{C}$ is closed under nonempty bounded unions, $X \in C$. In case $X=\emptyset$, we have that $\emptyset \in \mathrm{C}$.

This covers rows 2,6 , and 13 of the table, with or without rootedness. The case of binary conflict is similar, and we omit the details.

Next assume $\mathrm{C}$ is closed under $\bar{U}$. and with binary conflict. Let $\mathrm{E}=\left(E, \vdash_{1} \cup \vdash_{2} \cup \vdash_{3}\right)$ with

$$
\begin{aligned}
& X \vdash_{1} Y \text { iff }|Y| \leq 1, X \cap Y=\emptyset \text { and } X \cup Y \in C, \\
& X \vdash_{2} Y \text { iff } X=\emptyset,|Y|=2 \text { and } C o n(Y), \\
& X \vdash_{3} Y \text { iff } X=\emptyset \text { and }|Y|>2 \text {. }
\end{aligned}
$$


It is straightforward to check that

- $\mathrm{E}$ is always pure, singular and with binary conflict,

- if $\mathrm{C}$ is rooted, then so is $\mathrm{E}$,

- if $E$ is finite than $\mathrm{C}$ and $\mathrm{E}$ are trivially finitary, otherwise $\mathrm{C}$ and $\mathrm{E}$ can not be finitary,

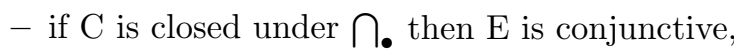

- and if $\mathrm{C}$ is $\bar{\bigcap}_{\text {. }}$-closed then $\mathrm{E}$ is locally conjunctive. We show that $\mathcal{L}(\mathrm{E})=\mathrm{C}$. That $C \subseteq L(\mathrm{E})$ goes exactly as in the previous case, so suppose $X \in \mathcal{L}(\mathrm{E})$. In case $|X| \leq 1$ there again is a $Z \subseteq X$ such that $Z \vdash X$, and we have $X=Z \cup X \in C$. So suppose $|X|>1$. For every $Y \subseteq X$ with $|Y|=2$ there is a $Z$ with $Z \vdash Y$. It must be that $Z=\emptyset$ and $\operatorname{Con}(Y)$. As $\mathrm{C}$ has binary conflict we have $\operatorname{Con}(X)$, i.e. $\exists W \in C$. $X \subseteq W$. Moreover, for any $e \in X$ there is a $Z_{e} \subseteq X$ such that $Z_{e} \vdash\{e\}$. By construction, $Z_{e} \cup\{e\} \in C$. As $\bigcup_{e \in X}\left(Z_{e} \cup\{e\}\right)=X \subseteq W \in C$, and $\mathrm{C}$ is closed under nonempty bounded unions, $X \in C$.

\subsection{Tying Petri nets}




\section{Event vs. configuration structures}

Definition 3.1 Let $\mathrm{C}=\langle E, C\rangle$ be a configuration structure. A set of events $X \subseteq E$ is consistent or conflict-free, written $C o n(X)$, if $\exists Z \in C . X \subseteq Z$. $X$ is finite-conflict-free, written $\operatorname{Con}_{f i n}(X)$, if

$$
\forall Y \subseteq_{\text {fin }} X . \operatorname{Con}(Y) .
$$

and binary-conflict-free, written $\mathrm{Con}_{2}(X)$, if

$$
\forall Y \subseteq X .(|Y| \leq 2 \Rightarrow \operatorname{Con}(Y)) .
$$

$\mathrm{C}$ is said to be closed under bounded unions $(\overline{\mathrm{U}})$ if

$$
X_{i} \in C(\text { for } i \in I) \wedge \operatorname{Con}\left(\bigcup_{i \in I} X_{i}\right) \Rightarrow \bigcup_{i \in I} X_{i} \in C,
$$

under finite-conflict-free unions $\left(\bar{\bigcup}^{f}\right)$ if

$$
X_{i} \in C(\text { for } i \in I) \wedge \operatorname{Con}_{f i n}\left(\bigcup_{i \in I} X_{i}\right) \Rightarrow \bigcup_{i \in I} X_{i} \in C
$$

and under binary-conflict-free unions $\left(\overline{\mathrm{U}}^{2}\right)$ if

$$
X_{i} \in C(\text { for } i \in I) \wedge \operatorname{Con}_{2}\left(\bigcup_{i \in I} X_{i}\right) \Rightarrow \bigcup_{i \in I} X_{i} \in C .
$$

$\mathrm{C}$ is closed under nonempty intersections $\left(\bigcap_{\bullet}\right)$ if

$$
X_{i} \in C(\text { for } i \in I \neq \emptyset) \Rightarrow \bigcap_{i \in I} X_{i} \in C
$$

and under bounded nonempty intersections $\left(\bar{\Pi}_{\bullet}\right)$ if

$X_{i} \in C($ for $i \in I \neq \emptyset) \wedge \operatorname{Con}\left(\bigcup_{i \in I} X_{i}\right) \Rightarrow \bigcap_{i \in I} X_{i} \in C$.

$\mathrm{C}$ has finite conflict if

$[\forall Y \subseteq X$. (Y finite $\Rightarrow \exists Z \in C . Y \subseteq Z \subseteq X)] \Rightarrow X \in C$ and binary conflict if

$[\forall Y \subseteq X .(|Y| \leq 2 \Rightarrow \exists Z \in C . Y \subseteq Z \subseteq X)] \Rightarrow X \in C$.

Theorem 4 Let $\mathrm{E}$ be an event structure.

- If $\mathrm{E}$ is conjunctive, then $L(\mathrm{E})$ is closed under $\bigcap$.

- If $\mathrm{E}$ is locally conjunctive, then $L(\mathrm{E})$ is $\bar{\bigcap}_{\mathbf{0}}$-closed.

- If $\mathrm{E}$ has finite conflict, then so does $L(\mathrm{E})$.

- If $\mathrm{E}$ has binary conflict, then so does $L(\mathrm{E})$.

- If $\mathrm{E}$ is singular, then $L(\mathrm{E})$ is closed under $\bar{U}$.

- If $\mathrm{E}$ is singular and with finite conflict, then $L(\mathrm{E})$ is closed under $\bar{U}^{f}$.

- If $\mathrm{E}$ is singular and with binary conflict, then $L(\mathrm{E})$ is closed under $\bar{U}^{2}$.

The same can be said for $S^{\infty}(\mathrm{E})$ and $S(\mathrm{E})$.

\begin{tabular}{|l|l|l|}
\hline $\begin{array}{l}\text { Event } \\
\text { structures }\end{array}$ & $\begin{array}{l}\text { Configuration } \\
\text { structures }\end{array}$ & $\begin{array}{l}\text { Propositional } \\
\text { theories }\end{array}$ \\
\hline \hline pure & - & pure \\
rooted & rooted & $(\geq 0$, any $)$ \\
conjunctive & closed under $\bigcap$. & $($ any, $\leq 1)$ \\
locally conj. & closed under $\bar{\bigcap}$. & (any, ddc) \\
finite confl. & finite conflict & (finite, any) \\
binary confl. & binary conflict & $(\leq 2$, any) \\
singular & closed under $\bar{\bigcup}$ & $(1$, any $),($ any, 0) \\
sing. \& fin. con. & closed under $\bar{\bigcup}^{f}$ & $(1$, any $),($ fin., 0$)$ \\
sing. \& bin. con. & closed under $\bar{\bigcup}^{2}$ & $(1$, any $),(\leq 2,0)$ \\
\hline
\end{tabular}

TABLE 2: Corresponding properties

Theorem 4, together with Proposition 1.5, is illustrated in the first two columns of Table 2. Here $\bigcap_{0}, \bar{\Pi}_{\bullet}, \bar{U}, \bar{U}^{f}$ and $\bar{U}^{2}$ stand for nonempty intersections, nonempty bounded intersections, bounded unions, finite-conflict-free unions and binary-conflictfree unions, respectively.

Note that a configuration structure that is closed under $\bar{U}^{f}$ is also closed under $\bar{U}$ and has finite conflict. Likewise, a configuration structure that is closed under $\bar{U}^{2}$ is also closed under $\bar{U}$ and has binary conflict. Now call a set of properties from the second column of Table 2 a package if it contains the property "closed under $\bar{\cup}^{f}$ " when it contains the properties "closed under U" and "having finite conflict", and it contains the property "closed under $\bar{U}^{2}$ " when it contains the properties "closed under U" and "having binary conflict".

Theorem 5 A configuration structure $\mathrm{C}$ has any package of properties from the second column of Table 2 iff there is an event structure $\mathrm{E}$ with the corresponding properties such that $\mathcal{L}(\mathrm{E})=\mathrm{C}$.

Proof: Let $\mathrm{C}=\langle E, C\rangle$ be a configuration structure. Define $\mathrm{E}=\langle E, \vdash\rangle$ by $X \vdash Y$ iff $X \cap Y=\emptyset \wedge X \cup Y \in C$. It is straightforward to check that

- $\mathrm{E}$ is always pure,

- if $\mathrm{C}$ is rooted, then so is $\mathrm{E}$,

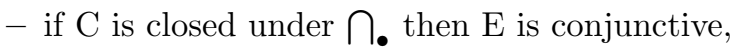

- and if $\mathrm{C}$ is $\overline{\mathrm{n}}_{\text {- }}$-closed then $\mathrm{E}$ is locally conjunctive. We show that $\mathcal{L}(\mathrm{E})=\mathrm{C}$. Suppose $x \in C$. For any $Y \subseteq x$ take $Z=x-Y$. Then $Z \subseteq x$ and $Z \vdash Y$. So $x \in \mathcal{L}(\mathrm{E})$. Conversely, suppose $X \in \mathcal{L}(\mathrm{E})$. Then there is a $Z \subseteq X$ such that $Z \vdash X$. By construction, $X=Z \cup X \in C$.

Next let $\mathrm{C}$ have finite conflict. Let $\mathrm{E}=\left\langle E, \vdash \cup \vdash^{\omega}\right\rangle$ with $\vdash$ defined as before, and $X \vdash^{\omega} Y$ iff $X=\emptyset$ and $Y$ infinite. It is straightforward to check that 
- $\mathrm{E}$ is always pure and with finite conflict,

- if $\mathrm{C}$ is rooted, then so is $\mathrm{E}$,

- if $\mathrm{C}$ is closed under $\bigcap_{\bullet}$ then $\mathrm{E}$ is conjunctive, no! and if $\mathrm{C}$ is $\bar{\bigcap}_{\text {. }}$-closed then $\mathrm{E}$ is locally conjunctive. We show that $\mathcal{L}(\mathrm{E})=\mathrm{C}$. That $C \subseteq L(\mathrm{E})$ goes exactly as in the previous case, so suppose $X \in \mathcal{L}(\mathrm{E})$. For any finite $Y \subseteq X$ there must be a $Z \subseteq X$ with $Z \vdash Y$. By construction, $Z \cup Y \in C$. As $Y \subseteq Z \cup Y \subseteq X$, and $\mathrm{C}$ has finite conflict, we have $X \in C$.

The case that $\mathrm{C}$ has binary conflict goes similarly.

Now assume $\mathrm{C}$ is closed under bounded unions $(\overline{\mathrm{U}})$.

Let $\mathrm{E}=\left\langle E, \vdash_{1} \cup \vdash_{2}\right\rangle$ with

$X \vdash_{1} Y$ iff $|Y|=1, X \cap Y=\emptyset$ and $X \cup Y \in C$,

$X \vdash_{2} Y$ iff $X=\emptyset,|Y| \neq 1$ and $\operatorname{Con}(Y)$.

It is straightforward to check that

- E is always pure and singular,

- if $\mathrm{C}$ is rooted, then so is $\mathrm{E}$,

- if $\mathrm{C}$ is closed under $\bigcap_{\text {. }}$ then $\mathrm{E}$ is conjunctive,

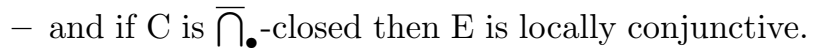
We show that $\mathcal{L}(\mathrm{E})=\mathrm{C}$. Suppose $x \in C$. For any $Y \subseteq x$ take $Z=x-Y$ if $|Y|=1$ and $Z=\emptyset$ otherwise. Then $Z \subseteq x$ and $Z \vdash Y$. So $x \in \mathcal{L}(\mathrm{E})$. Conversely, suppose $X \in \mathcal{L}(\mathrm{E})$. Then there is a $Z \subseteq X$ such that $Z \vdash X$. In case $|X|=1$ we have $X=Z \cup X \in C$. In case $|X| \neq 1$ it must be that $Z=\emptyset$ and $\operatorname{Con}(X)$. Moreover, for any $e \in X$ there is a $Z_{e} \subseteq X$ such that $Z_{e} \vdash\{e\}$. By construction, $Z_{e} \cup\{e\} \in C$. As $\bigcup_{e \in X}\left(Z_{e} \cup\{e\}\right)=X$ and $\operatorname{Con}(X)$, and $\mathrm{C}$ is closed under bounded unions, $X \in C$.

Next assume $\mathrm{C}$ is closed under $\bar{\cup}^{2}$.

Let $\mathrm{E}=\left\langle E, \vdash_{1} \cup \vdash_{2} \cup \vdash_{3}\right\rangle$ with

$X \vdash_{1} Y$ iff $|Y|=1, X \cap Y=\emptyset$ and $X \cup Y \in C$,

$X \vdash_{2} Y$ iff $X=\emptyset,(|Y|=0$ or $|Y|=2)$ and $\operatorname{Con}(Y)$,

$X \vdash_{3} Y$ iff $X=\emptyset$ and $|Y|>2$.

It is straightforward to check that

- E is always pure, singular and with binary conflict,

- if $\mathrm{C}$ is rooted, then so is $\mathrm{E}$,

- if $\mathrm{C}$ is closed under $\bigcap_{\text {. }}$ then $\mathrm{E}$ is conjunctive,

no! and if $\mathrm{C}$ is $\bar{\bigcap}_{\text {. }}$-closed then $\mathrm{E}$ is locally conjunctive. We show that $\mathcal{L}(\mathrm{E})=\mathrm{C}$. That $C \subseteq L(\mathrm{E})$ goes exactly as in the previous case, so suppose $X \in \mathcal{L}(\mathrm{E})$. In case $|X|=1$ there again is a $Z \subseteq X$ such that $Z \vdash X$, and we have $X=Z \cup X \in C$. So suppose $|X| \neq 1$. For every $Y \subseteq X$ with $|Y|=0$ or $|Y|=2$ there is a $Z$ with $Z \vdash Y$. It must be that $Z=\emptyset$ and $\operatorname{Con}(Y)$. Hence $\mathrm{Con}_{2}(X)$. Moreover, for any $e \in X$ there is a $Z_{e} \subseteq X$ such that $Z_{e} \vdash\{e\}$. By construction, $Z_{e} \cup\{e\} \in C$. As $\cup_{e \in X}\left(Z_{e} \cup\{e\}\right)=X$ and $\operatorname{Con}_{2}(X)$, and $\mathrm{C}$ is closed under binary-conflict-free unions, $X \in C$.

The case that $\mathrm{C}$ is closed under $\overline{\mathrm{U}}^{f}$ goes likewise.

\subsection{Axiomatisation of set systems}

In this section we consider set systems $\mathrm{C}=\langle E, C\rangle$ from a logical point of view: $E$ is thought of as a collection of propositions and $C$ as the collection of models. Connecting with the computational point of view, we associate with an event the proposition that it has happened. There is an associated theory $T(C)$ of all valid sentences, those holding in all models; these are the laws of $\mathrm{C}$.

To make this precise, we choose a language: infinitary propositional logic with $E$ as the set of propositional variables, and closed under $\neg$ (negation) and $\bigwedge$ (conjunction of sets of formulae). We make free use of other standard connectives such as $\Rightarrow, \bigvee, \perp, \top$ : they are all definable. A formula $\phi$ is valid in $\langle E, C\rangle$ iff it is true in all models, that is, elements of $C ; T(\mathrm{C})$ denotes the class of formulae valid in C. Equally, given a class $T$ of formulae over a set $E$, we can define a set system $\mathrm{C}(T)=\langle E, \mathcal{M}(T)\rangle$, where $\mathcal{M}(T)$ is the set of models of $T$, those interpretations making every formula in $T$ true. We say that $T$ axiomatises $\mathrm{C}(T)$. In particular $T(C)$ axiomatises $\mathrm{C}$ for any set system $\mathrm{C}$.

This point of view is due to Pratt $[11,21]$. He noted a natural "conjunctive normal form." For any two subsets $X, Y$ of $E$, let the clause $X \Rightarrow Y$ abbreviate the implication $\bigwedge X \Rightarrow \bigvee Y$; we say that the elements of $X$ are the antecedents of the clause, and those of $Y$ its consequents. Then for any $\langle E, C\rangle$, if $T$ is the collection of clauses valid in $\langle E, C\rangle$ we have $\langle E, C\rangle=\mathrm{C}(T)$. Thus any set system can be axiomatised by a set of clauses.

We now consider correspondences between axiomatisations by classes of formulae and closure conditions on set systems.

First, there is logical interest in Horn clauses where there are finitely many antecedents and one consequent, and also in Scott clauses where, more generally, there may be finitely many consequents [3, 22].

Proposition 3.1 A set system $\langle E, C\rangle$ is Horn clause axiomatisable iff $C$ is closed under intersections and directed unions. It is Scott clause axiomatisable iff $C$ is closed in the product topology on $2^{E}$.

Proof: For the first part, the implication from left to right is easy to see. For the converse, we refer forward to the fifth entry in Table 3.1 where we have an axiomatisation by clauses of the form $X \Rightarrow Y$ where $Y$ is empty or a singleton. But the first case cannot obtain, as here $E$ is a model.

For the second part, the product topology on $2^{E}$ is the $E$-fold power of the discrete topology on the twopoint set. It has as basis all sets of the form $\mathcal{U}_{x, y}=$ $\{m \subseteq E \mid x \subseteq m,(m \cap y)=\emptyset\}$ where $x, y$ are finite 
subsets of $E$. The first part now follows, noting that the complement of $\mathcal{U}_{x, y}$ is the set of models of $x \Rightarrow y$.

Next we consider closure conditions that arise naturally when considering configuration structures. We denote closure under non-empty intersections by $\bigcap_{\bullet}$, under bounded non-empty intersections by $\bar{\bigcap}_{\bullet}$, under bounded non-empty unions by $\bar{U}$ and under directed unions by $U_{\uparrow}$. The results appear below. The

\begin{tabular}{|c|c|c|c|}
\hline stable & $\begin{array}{l}\bar{\bigcap}_{\bullet}, \bar{U} \\
\bar{\bigcap}_{\bullet}, U_{\uparrow}, \bar{U}\end{array}$ & $\begin{array}{l}\equiv \\
\Leftarrow \\
\equiv \\
\Leftarrow\end{array}$ & $\begin{array}{l}\text { (any, ddc) } \\
\text { (finite, ddfc) } \\
(\leq 1, \text { ddc }) \\
(\leq 1, \text { ddfc })\end{array}$ \\
\hline prime & $\begin{array}{l}\bigcap_{\bullet}, U, \bar{U} \\
\bigcap_{\bullet}, \cup_{\uparrow}, \bar{U}\end{array}$ & $\begin{array}{l}\equiv \\
\equiv \\
\equiv \\
\equiv\end{array}$ & $\begin{array}{l}(\text { any }, \leq 1) \\
(\text { finite }, \leq 1) \\
(\leq 1,1),(\text { any }, 0) \\
(\leq 1,1),(\text { finite }, 0)\end{array}$ \\
\hline union & $\underset{\bigcup_{\uparrow}}{\bar{U}, U_{\uparrow}}$ & $\begin{array}{l}\equiv \\
\Leftarrow \\
\Leftarrow\end{array}$ & $\begin{array}{l}(\leq 1, \text { any }),(\text { any }, 0) \\
(\leq 1, \text { any }),(\text { finite }, 0) \\
\text { (finite, any) }\end{array}$ \\
\hline
\end{tabular}

last column indicates the form of the allowed formulae which are all implications. For example: $($ any, $\leq 1)$ indicates a clause with no restriction on its antecedent and whose consequent has at most one element; and (any, ddc) indicates an implication whose premise can be any conjunction of propositional letters, and whose conclusion has the "ddc" form. This is a formula $\vee_{j \in J} \wedge Z_{j}$ where the $Z_{j}$ are sets of letters, and we write $\bigvee \Phi$ for $(\bigvee \Phi) \wedge \bigwedge\left\{\neg\left(\phi \wedge \phi^{\prime}\right) \mid \phi, \phi^{\prime} \in \Phi, \phi \neq \phi^{\prime}\right\}$, the disjoint disjunction of $\Phi$. In case all the $Z_{j}$ are finite, we have the "ddfc" form. So, for example the sixth entry states that a configuration structure is axiomatisable by clauses of the form (finite, $\leq 1$ ) iff it is closed under non-empty bounded intersections and directed unions; this is essentially due to Larsen and Winskel [14] as axiomatisations of the form (finite, $\leq 1$ ) correspond to Scott information systems. The entries with $\Leftarrow$ 's indicate that only the implication from right to left holds.

We now show the validity of all entries of Table 3.1. The implications from right to left are straightforward. As an example we do the first one, leaving the rest to the reader. To this end we show that the collection of models of a family of implications of the form $\bigwedge X \Rightarrow \vee_{j \in J} \wedge Z_{j}$ is closed under bounded non-empty intersections. Suppose that $\left\{m_{i} \mid i \in I\right\}$ is such a collection, with upper bound $m^{\prime}$. Let $m$ be the intersection of the $m_{i}$; we must show it is a model. To this end, choose one implication of the above form and suppose that $m$ includes its premise $X$. Then so does $m^{\prime}$ and hence there is a unique $j$ in $J$ such that $Z_{j} \subseteq m^{\prime}$; but since, for any $i$ in $I, m_{i}$ is a model with $X \subseteq m_{i} \subseteq m^{\prime}$ we have that $Z_{j} \subseteq m_{i}$. So $Z_{j} \subseteq m$, and this must be the unique such $j$ as $m \subseteq m^{\prime}$, since $I$ is non-empty.

Next, for a counterexample to the implications from left to right for the second, fourth and the last two entries, take the collection of all co-singleton proper subsets of the natural numbers. This is closed under non-empty bounded meets, non-empty bounded unions and directed unions. However any valid formula of any of the forms (finite, ddfc), (finite, any) or $(\leq 1$, any) holds also of the set of all natural numbers.

We now consider the converse implications, considering a configuration structure $\mathrm{C}=\langle E, C\rangle$ and showing that various closure conditions on $C$ yield corresponding axiomatisations of $\mathrm{C}$. We begin with the first "stability" entry, and suppose $C$ closed under bounded non-empty intersections. For any $X \subseteq E$, consider the implication $\phi_{X}=\bigwedge X \Rightarrow \bigvee_{j \in J_{X}} \wedge \gamma_{j}$, where $\gamma_{j}$ $\left(j \in J_{X}\right)$ is a $1-1$ indexing of all minimal elements of $C$ that contain $X$. Since $C$ is closed under non-empty bounded intersections, every element of $C$ that contains $X$ contains a unique $\gamma_{j}$ (see ???). This means that the implications are valid in $C$. So it is enough to show that any model $m$ of these formulae is in $C$. Now $m$ is a model of $\phi_{m}$ and so for some $j$ we have $m \supseteq \gamma_{j}$. But $\gamma_{j} \supseteq m$, so $m$ is in $C$, as required.

In the third entry, we must show that $\mathrm{C}$ is axiomatised by the implications $\phi_{x}$ where $x$ is empty or a singleton, together with the valid clauses of the form $X \Rightarrow \emptyset$. The conclusion will then follow, as $X \Rightarrow \emptyset$ is equivalent to $\top \Rightarrow(\bigwedge X) \bullet \top$.

So suppose that $m$ is a model of these formulae; we will show it is a configuration. First, $m \Rightarrow \emptyset$ cannot be valid in $\mathrm{C}$, and so $m$ has an upper bound $\gamma$ in $C$. Next, in case $m$ is empty, considering $\phi_{\emptyset}$ we see that it is in $C$. Otherwise, for each $e$ in $m$, considering $\phi_{\{e\}}$ we obtain a configuration $\gamma_{e}$ in $C$ such that $e \in \gamma_{e} \subseteq m \subseteq \gamma$. But now we have that $\gamma_{e}(e \in m)$ is a non-empty bounded collection of configurations, and so its union $m$ is a configuration, as required.

Let us now turn to the "prime" entries where closure under non-empty intersections is always assumed. In that case if there is a configuration containing a set $X \subseteq C$, there is a least such configuration, $\gamma_{X}$. We now show that in the first such entry, $\mathrm{C}$ is axiomatised by the set of valid clauses of the form (any, $\leq 1)$. Let $m$ be a model of this set; we have only to show that it is a configuration. Since $m$ does not satisfy the clause $m \Rightarrow \emptyset$, that cannot be valid; so there is a configuration containing $m$ and $\gamma_{m}$ exists. Now if $e$ is in $\gamma_{m}$ then 
$m \Rightarrow e$ is valid (by the definition of $\gamma_{m}$ ); so $e$ is in $m$. We therefore have that $m$ is a configuration, being $\gamma_{m}$.

For the next entry, it is also assumed that $C$ is closed under directed unions. Let us prove that if a clause $X \Rightarrow Y$ is valid, then so is $x \Rightarrow Y$ for some finite subset $x$ of $X$. (The result will then follow from the previous axiomatisability result.) Suppose, for the sake of contradiction, that no such $x \Rightarrow Y$ is valid. Then if $x \subseteq_{\text {fin }} X$ there is a configuration containing $x$ but with empty intersection with $Y$. So $\gamma_{x}$ exists and $\gamma_{x} \cap Y=\emptyset$. Further, by the definition of $\gamma_{x}$, we have that if $x \subseteq y \subseteq$ fin $X$ then $\gamma_{x} \subseteq \gamma_{y}$. But then, by the assumption of the closure of $C$ under directed unions, $\bigcup\left\{\gamma_{x} \mid x \subseteq_{\text {fin }} X\right\}$ is a configuration not satisfying $X \Rightarrow Y$, providing the required contradiction.

We now show that if $C$ is closed under bounded, non-empty unions (but not necessarily under directed unions) then if a clause of the form $X \Rightarrow Y$ is valid, with $Y$ empty or a singleton, then either $X \Rightarrow \emptyset$ is valid, or $x \Rightarrow Y$ is valid, with $x \subseteq X$ empty or a singleton. Together with the previous result on closure under directed unions, this will establish the correctness of the third and fourth entries.

So, consider $X \Rightarrow Y$. If either $X \Rightarrow \emptyset$ or $\emptyset \Rightarrow Y$ are valid, we are done. Otherwise, $Y$ is a singleton, there is a configuration containing $X$, and $X$ is non-empty. Suppose now, for the sake of contradiction, that no $e \Rightarrow Y$ is valid, with $e$ in $X$. Then for $e \in X$ there is a configuration containing $e$ and with empty intersection with $Y$; so $\gamma_{\{e\}}$ is also such a configuration, and it is contained in $\gamma_{X}$. But now, by the assumption of closure of non-empty bounded unions, $\bigcup\left\{\gamma_{\{e\}} \mid e \in\right.$ $X\}$ is a configuration not satisfying $X \Rightarrow Y$, providing the required contradiction.

Turning to the "union" entries, we have only to consider the case where $C$ is closed under non-empty bounded unions. We show that it is axiomatised by all valid formulae of any of the forms $e \Rightarrow \bigvee_{\lambda \in \Lambda} \wedge X_{\lambda}$, or $\bigwedge X \Rightarrow \perp$ or else $\top \Rightarrow \bigvee E$. The result then follows by expanding out the formulae of the first kind into disjunctive normal form.

Let $m$ be a model of these valid formulae. If it is empty, then it does not satisfy $T \Rightarrow \bigvee E$; so some configuration also does not satisfy this formula. That configuration must also be $\emptyset$ and so equal to $m$. Otherwise, we can assume that $m$ is non-empty. Now, as $m$ does not model $\bigwedge m \Rightarrow \perp$, that formula cannot be valid, and so $m$ is contained in some configuration, $\gamma$. Next consider the formulae:

$$
e \Rightarrow \bigvee_{\lambda \in \Lambda_{e}} \bigwedge \gamma_{\lambda}
$$

for $e \in m$, where $\gamma_{\lambda}$ runs through the configurations containing $e$. As these are evidently valid, $m$ is a model of them, and so we find for each $e \in m$ a configuration $\gamma_{e} \subseteq m(\subseteq \gamma)$. But then, by the assumed closure condition, $m=\bigcup_{e \in m} \gamma_{e}$ is itself a configuration. With this, we have established the validity of all entries in Table 3.1.

A minor, but useful, variation on these results is to restrict to rooted configuration structures (those containing $\emptyset$ ); then in the last column one changes "any" to "non-empty," and "finite" to "finite and nonempty" and " $\leq 1$ " to " 1 " on the left of the implications (but not the right). Further in the ???

We now consider axiomatisations of event structures. As the set-theoretic characterisation of event structures involves other than closure conditions finiteness and coincidence-freeness, we do not expect a precise result. Say that $T$ axiomatises a configuration structure $\mathrm{C}$ up to its standard part iff the standard parts of $\mathrm{C}$ and $\mathrm{C}(T)$ are event isomorphic. The results are given in Table 3; they hold both with and without the parenthetical conjunctions; it is assumed that all configuration structures involved contain $\emptyset$. For example, the second row asserts that, up to standard parts, the configuration structures of general event structures are those containing $\emptyset$ and closed under non-empty bounded unions (and directed unions), and are axiomatised by formulae of one of the two forms: (1, any) or (finite and non-empty, 0 ). The first row suggests the intriguing possibility of a yet more general form of event structure, corresponding to arbitrary configuration structures. 
TABLE 3: Standard part characterisations

\begin{tabular}{|l|l|l|}
\hline unknown & $\left(\bigcup_{\uparrow}\right)$ & (finite and non-empty, any) \\
\hline general & $\bar{U}\left(\right.$ and $\left.\bigcup_{\uparrow}\right)$ & $(1$, any $),($ finite and non-empty, 0$)$ \\
\hline stable & $\bar{U}, \bar{\bigcap}_{\text {. }}\left(\right.$ and $\left.\bigcup_{\uparrow}\right)$ & $(1$, ddc $),($ finite and non-empty, 0$)$ \\
\hline prime & $\bar{U}, \bigcap_{\bullet}\left(\right.$ and $\left.\bigcup_{\uparrow}\right)$ & $(1,1),($ finite and non-empty, 0$)$ \\
\hline
\end{tabular}

\section{Petri nets}

\section{Definition 4.1}

A labelled Petri net is a tuple $\langle S, T, F, I, l\rangle$ with $\langle S, T . F, I\rangle$ a net and $l: T \rightarrow$ Act, the labelling function.

Labelled Petri nets are pictured by inscribing the label of a transition in its corresponding box.

Definition 4.2 A net is said to be without arcweights if the range of $F$ is $\{0,1\}$. It is safe if all reachable markings are plain sets (as opposed to multisets) and every transition has at least one preplace.

\subsection{Unbounded parallelism}

It is tempting to generalise the firing rule of Definition 1.12 to infinite multisets. The simplest implementation of this idea, however, yields infinitary markings, as illustrated in Figure 1. After all transitions $t_{i}$
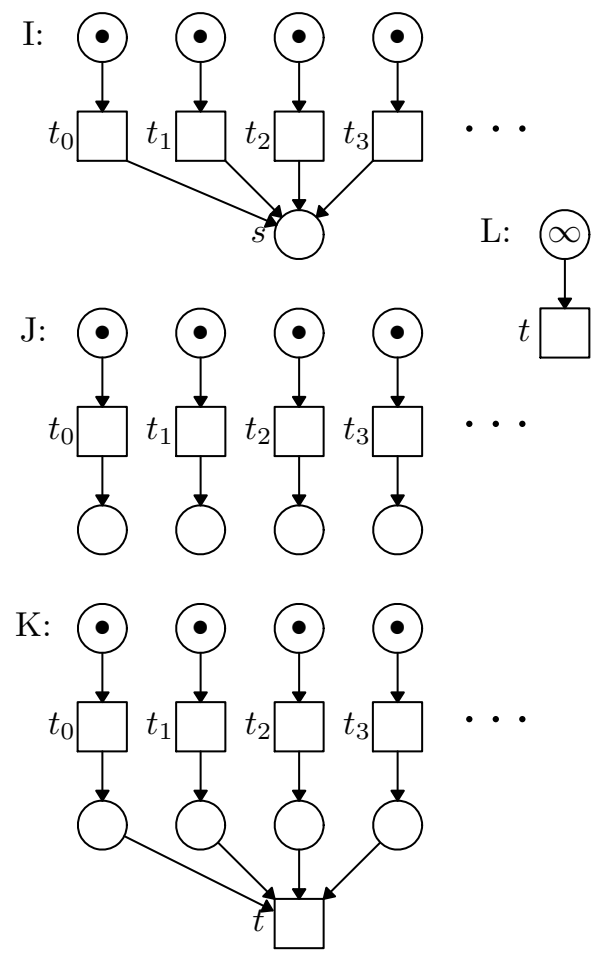

Figure 1: Unbounded parallelism

$(i \in \mathbb{N})$ of net I have fired (in one step) there are infinitely many tokens in place $s$, contrary to the definition of a marking. One way to fix this problem is to allow infinite markings. This, however, causes the problem illustrated by the net L: after transition $t$ has fired countably often, are there tokens left to fire once more? Such problems are best avoided by sticking to finitary markings. Another solution is to allow a multiset of transitions to fire only if by doing so none of its postplaces receives an infinite amount of tokens. This would enable any finite multiset over $\left\{t_{i} \mid i \in \mathbb{N}\right\}$ to fire initially, but no infinite one. A disadvantage of this solution is that the nets I and J, which normally would be regarded equivalent, have now a different behaviour, as in $\mathrm{J}$ all transitions $t_{i}$ can still fire in one step. As a consequence, our forthcoming theorem that any net is step bisimulation equivalent to a safe net, or a prime event structure, would no longer hold; I constitutes a counterexample.

Therefore we stick in this paper to the convention, formalised by Definition 1.12, that only finitely many transitions can fire in a finite time. As a consequence, the transition $t$ in net $\mathrm{K}$ can never fire and this net is semantically equivalent to I and J.

\section{$4.2 \quad$ Self-concurrency}

In older papers on Petri nets a multiset of transitions was allowed to fire only if it was a set, i.e., no transition could fire multiple times concurrent with itself. The argument for this restriction was that a transition could be thought of as a subsystem like a printer, that can only print one file at a time. When there are enough tokens in its preplaces (representing printrequests and other preconditions for printing) to handle two files, these have to be printed one by one. This argument has been convincingly rebutted in GOLTz \& REISIG [8], and since then multisets are generally allowed to fire. In any case, the behaviour of nets under the self-sequential firing rule can easily be encoded into the behaviour of nets under the self-concurrent firing rule of Definition 1.12 by the following proposition.

Proposition 4.1 Any net $\mathrm{N}$ can be transformed into a net $\mathrm{N}^{\prime}$ such that 
- under the self-sequential firing rule $\mathrm{N}^{\prime}$ behaves the same as $\mathrm{N}$,

- in $\mathrm{N}^{\prime}$ we have $M \stackrel{U}{\longrightarrow} M^{\prime}$ only if $U$ is a set.

Proof: For any transition $t$ in $\mathrm{N}$ add a self-loop, consisting of a place $s_{t}$ with $I\left(s_{t}\right)=F\left(s_{t}, t\right)=F\left(t, s_{t}\right)=1$ and $\forall u \neq t . F\left(s_{t}, u\right)=F\left(u, s_{t}\right)=0$. This yields the required net $\mathrm{N}^{\prime}$.

Further on we assume the firing rule of Definition 1.12, but indicate when necessary what has to be changed in case the self-sequential firing rule is assumed.

\subsection{Individual vs. collective tokens}

There are two different schools of thought in interpreting the causal behaviour of nets, which can be described as the individual and collective token philosophy. ${ }^{1}$ The following example illustrates their difference.

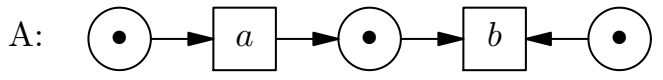

In this net, the transitions labelled $a$ and $b$ can fire once each. After $a$ has fired, there are two tokens in the middle place. According to the individual token philosophy, it makes a difference which of these tokens is used in firing $b$. If the token that was there already is used (which must certainly be the case if $b$ happens before the token from $a$ arrives), the transitions $a$ and $b$ are causally independent. If the token that was produced by $a$ is used, $b$ is causally dependent on $a$. Thus, the net A above has two maximal computations, that can be characterised by the partial orders ${ }_{b}^{a}$ and $a \rightarrow b$. According to the collective token philosophy on the other hand, all that is present in the middle place after the occurrence of $a$ is the number 2. The preconditions for $b$ to fire do not change, and consequently $b$ is always causally independent of $a$.

The individual token approach has been formalised by the notion of a process, described in GOLTZ \& REISIG [8]. A causality respecting bisimulation relation based on this approach was proposed by BEST, Devillers, Kiehn \& Pomello [1] under the name fully concurrent bisimulation. Below we contribute a few rather fine equivalences based on the collective token philosophy. That both philosophies yield incomparable notions of equivalence follows from the following example.

\footnotetext{
${ }^{1}$ The individual token interpretation of ordinary nets should not be confused with the concept of Petri nets with individual tokens; there the individuality is hardwired into the syntax of nets.
}

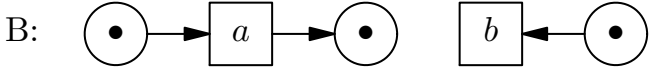

In the collective token philosophy the precondition of $b$ expressed by the place in the middle is redundant, and hence $\mathrm{A}$ must be equivalent to B. A and B are not fully concurrent bisimulation equivalent however, as B lacks the computation $a \rightarrow b$. On the other hand, $\mathrm{A}$ is fully concurrent bisimulation equivalent with $\mathrm{C}$ below.

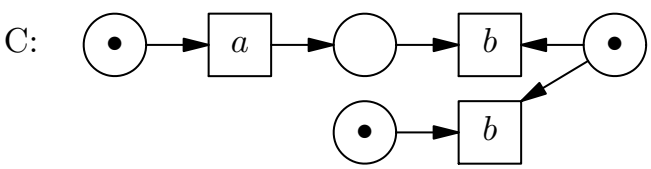

In fact, $\mathrm{C}$ is the occurrence net obtained from $\mathrm{A}$ by the unfolding of Meseguer, Montanari \& SAssone [15], mentioned in the introduction. In the individual token philosophy, both nets have the computations $a \rightarrow b$ and $a$. However, A does not have a run $a \rightarrow b$ in the collective token philosophy, and can therefore not be equivalent to $\mathrm{C}$ in any causality preserving way.

\subsection{1-Unfolding}

Below we show that the restriction to 1-occurrence nets is not very crucial; every net can be "unfolded" into a 1-occurrence net without changing its behaviour in any essential way. However, the unfolding cannot be configuration equivalent to the original, as the identity of transitions cannot be preserved.

Definition 4.3 Let $N=\langle S, T, F, K, I, l\rangle$ be a Petri net. Its 1-unfolding $N^{\prime}=\left\langle S^{\prime}, T^{\prime}, F^{\prime}, K^{\prime}, I^{\prime}, l^{\prime}\right\rangle$ into a 1-occurrence net is given by (for $s \in S, t \in T, u \in T^{\prime}$ )

- $T^{\prime}=T \times \mathbb{N}$ and $l^{\prime}((t, n))=l(t)$,

- $S^{\prime}=S \cup\left(T^{\prime} \times\{*\}\right)$,

- $F^{\prime}(s,(t, n))=F(s, t)$ and $F^{\prime}((t, n), s)=F(t, s)$,

- $F^{\prime}(u,(u, *))=F^{\prime}\left((u, *), u^{\prime}\right)=F\left(u^{\prime},(u, *)\right)=0$ and $F^{\prime}((u, *), u)=1$ for $u, u^{\prime} \in T^{\prime}$ with $u \neq u^{\prime}$,

- $K^{\prime}(s)=K(s)$ and $K((u, *))=\infty$,

- $I^{\prime}(s)=I(s)$ and $I^{\prime}((u, *))=1$.

Thus, every transition is replaced by countably many copies, each of which is connected with its environment (though the flow relation) in exactly the same way as the original. Furthermore, for every such copy $u$ an extra place $(u, *)$ is created, containing one initial token, and having no incoming arcs and only one outgoing arc, going to $u$. This place guarantees that $u$ can fire only once. In any reachable marking of the unfolded net, one can see exactly which transitions have fired, namely those transitions $u$ for which the place 
$(u, *)$ is empty. Hence every such marking has only one configuration.

Note that the construction in Definition 4.3 does not introduce self-loops. Thus unfoldings of pure nets remain pure.

It is possible to give a slightly different interpretation of nets, namely by excluding transitions from firing concurrently with themselves. ${ }^{2}$ This amounts to simplifying Definition 1.12 by requiring $U$ to be a set rather than a multiset. Under this interpretation our unfolding could introduce concurrency that was not present before. However, for this purpose Definition 4.3 can be adapted by removing the initial tokens from the places $((t, n), *)$ for $t \in T$ and $n>0$ (but leaving the token in $((t, 0), *))$, and adding an arc from transition $(t, n)$ to place $((t, n+1), *)$ for every $t \in T$ and $n \in \mathbb{N}$.

\section{References}

[1] E. Best, R. Devillers, A. Kiehn \& L. Pomello (1991): Concurrent bisimulations in Petri nets. Acta Informatica 28, pp. 231-264.

[2] G. Boudol (1990): Flow event structures and flow nets. In I. Guessarian, editor: Semantics of Systems of Concurrent Processes, Proceedings LITP Spring School on Theoretical Computer Science, La Roche Posay, France, LNCS 469, Springer-Verlag, pp. 6295.

[3] D.M. GabBay (1981): Semantic Investigations in Heyting's Intuitionistic Logic, Synthese Library 148. D. Reidel.

[4] R.J. van GlabBeek (1995): History preserving process graphs. Report, Stanford University, Available at ftp://boole.stanford.edu/pub/DVI/history.dvi.gz.

[5] R.J. van Glabbeek \& U. Goltz (1989): Equivalence notions for concurrent systems and refinement of actions. In A. Kreczmar \& G. Mirkowska, editors: Proceedings $14^{\text {th }}$ Symposium on Mathematical Foundations of Computer Science, PorąbkaKozubnik, Poland 1989, LNCS 379, Springer-Verlag, pp. 237-248.

[6] R.J. van Glabbeek \& U. Goltz (1990): Refinement of actions in causality based models. In J.W. de Bakker, W.P. de Roever \& G. Rozenberg, editors: Proceedings REX Workshop on Stepwise Refinement of Distributed Systems: Models, Formalism, Correctness, Mook, The Netherlands, May/June 1989, LNCS 430, Springer-Verlag, pp. 267-300.

\footnotetext{
${ }^{2}$ This distinction is independent of the individual-collective token dichotomy, thus yielding four computational interpretations of nets.
}

[7] R.J. van Glabbeek \& F.W. Vaandrager (1987): Petri net models for algebraic theories of concurrency. In J.W. de Bakker, A.J. Nijman \& P.C. Treleaven, editors: Proceedings PARLE conference, Eindhoven, Vol. II (Parallel Languages), LNCS 259, SpringerVerlag, pp. 224-242.

[8] U. Goltz \& W. Reisig (1983): The non-sequential behaviour of Petri nets. Information and Computation 57, pp. 125-147.

[9] J. Gunawardena (1992): Causal automata. Theoretical Computer Science 101, pp. 265-288.

[10] V. Gupta (1994): Chu Spaces: A Model of Concurrency. $\mathrm{PhD}$ thesis, Stanford University. Available at ftp://boole.stanford.edu/pub/gupthes.ps.gz.

[11] V. Gupta \& V.R. Pratt (1993): Gates accept concurrent behavior. In Proc. 34th Ann. IEEE Symp. on Foundations of Comp. Sci., pp. 62-71.

[12] P.W. Hoogers, H.C.M. Kleijn \& P.S. ThiaGARAJan (1993): Local event structures and Petri nets. In E. Best, editor: Proceedings CONCUR 93, Hildesheim, Germany, LNCS 715, Springer-Verlag, pp. 462-476.

[13] R. LANGERAK (1992): Transformations and Semantics for LOTOS. PhD thesis, Department of Computer Science, University of Twente.

[14] K.G. Larsen \& G. Winskel (1991): Using information systems to solve recursive domain equations. Information and Computation 91(2), pp. 232-258.

[15] J. Meseguer, U. Montanari \& V. Sassone (1992): On the semantics of Petri nets. In W.R. Cleaveland, editor: Proceedings CONCUR 92, Stony Brook, NY, USA, LNCS 630, Springer-Verlag, pp. 286-301.

[16] R. Milner (1980): A Calculus of Communicating Systems, LNCS 92. Springer-Verlag.

[17] M. Nielsen, G.D. Plotkin \& G. Winskel (1981): Petri nets, event structures and domains, part I. Theoretical Computer Science 13(1), pp. 85-108.

[18] G.M. Pinna \& A. Poigné (1995): On the nature of events: another perspective in concurrency. Theoretical Computer Science 138(2), pp. 425-454.

[19] G.D. Plotkin \& V.R. Pratt (1988): Teams can see pomsets. Manuscript available at ftp://boole.stanford.edu/pub/DVI/pp2.dvi.gz.

[20] V.R. PRATt (1991): Modeling concurrency with geometry. In Proc. 18th Ann. ACM Symposium on Principles of Programming Languages, pp. 311-322. 
[21] V.R. PRATt (1994): Chu spaces: complementarity and uncertainty in rational mechanics. Tech. report, TEMPUS Summer School, Budapest. Available at ftp://boole.stanford.edu/pub/DVI/bud.dvi.gz.

[22] D.S. ScotT (1974): Completeness and axiomatizability in many-valued logic. In L. Henkin et al., editors: Proc. Tarski Symposium, AMS, pp. 411-435.

[23] G. Winskel (1987): Event structures. In W. Brauer, W. Reisig \& G. Rozenberg, editors: Petri Nets: Applications and Relationships to Other Models of Concurrency, Advances in Petri Nets 1986, Part II; Proceedings of an Advanced Course, Bad Honnef, September 1986, LNCS 255, Springer-Verlag, pp. 325-392.

[24] G. Winskel (1989): An introduction to event structures. In J.W. de Bakker, W.P. de Roever \& G. Rozenberg, editors: REX School/Workshop on Linear Time, Branching Time and Partial Order in Logics and Models for Concurrency, Noordwijkerhout, The Netherlands, May/June 1988, LNCS 354, Springer-Verlag, pp. 364-397.

A topic for future research will be to reconcile the two interpretations by adapting the definitions of $\mathcal{S}, \mathcal{L}$ and $\mathcal{R}$ for impure event structures - without touching the pure case -in such a way that $\mathcal{S}=\mathcal{R} \circ \mathcal{L}$ holds everywhere. This will involve extending the universe of configuration structures as well. When this is done, the current ones will be called pure.

\section{From previous intro}

Formulae In Section 3.1 we consider set systems from the point of view of (infinitary) propositional logic: $E$ is now thought of as the set of propositions and $C$ as the set of models. Following PRATT [21] we observe a bijective correspondence between configuration structures and infinitary propositional theories up to logical equivalence. We give a number of results equating the closure of $C$ under certain operations with its axiomatisation by formulae of certain simple forms.

The left-closed interpretation corresponds with a logical view of event structures as propositional theories. A somewhat different logical view was presented in GunaWARDEnA [9]. The precise relations with ours are yet to be investigated.

Petri nets In Meseguer, Montanari \& Sassone [15], arbitrary (non-safe) Petri nets are unfolded into occurrence nets. Their unfolding generalises the one of [17] and preserves the behaviour of nets under a particular interpretation due to GoLTz \& ReIsig [8]. We call this interpretation the individual token interpretation. It follows that under the individual token interpretation the behaviour of any Petri net can be repre- sented by a prime event structure. An alternative way of understanding the behaviour of nets is the collective token interpretation. In the latter view there are nets which cannot be represented by an event structure, let alone by a prime one with binary conflict, or an occurrence net.

In Section 4 we establish a connection between pure nets and configuration structures. Pure nets are nets without self-loops. We 1-unfold pure nets into pure 1occurrence nets, which generalise the occurrence nets of [17]. These pure 1-occurrence nets are shown to correspond with rooted finitary configuration structures through translations in both directions. The translation from configuration structures to nets passes through the propositional representation of configuration structures. These translations preserve the identity of events and configurations, i.e. translating a configuration structure to a net and back results in the original configuration structure. Information is lost in the translation of pure 1-occurrence nets to configuration structures, but we argue that the behaviour of nets expressible in terms of action occurrences and the causal relationships between them - under the collective token interpretation - is preserved. Although the translations can easily be extended to nets with selfloops, the latter argument would no longer apply.

In future work we plan to extend our models and translations to match the expressive power of arbitrary nets with self-loops. We also would like to connect our models with appropriate versions of higher dimensional automata [20].

Acknowledgment We thank Vladimiro Sassone for helpful comments. 